\title{
New directions for structural and functional neuroimaging markers in migraine
}

\author{
Ph.D. thesis \\ Dániel Veréb, MD \\ Clinical and Experimental Neuroscience Program \\ Doctoral School of Clinical Medicine \\ Faculty of Medicine, University of Szeged
}

Supervisor: Zsigmond Tamás Kincses, MD, PhD, DSc

Department of Radiology, Albert Szent-Györgyi Clinical Center

University of Szeged

Szeged

2020 


\section{Contents}

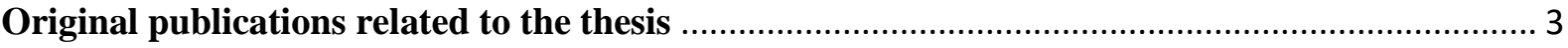

Original publications not directly related to the thesis ............................................................. 3

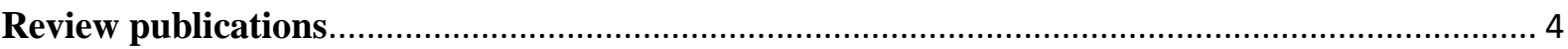

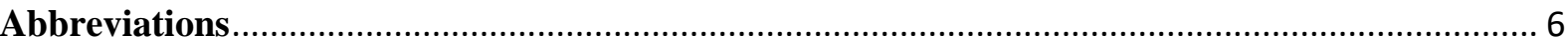

Summary

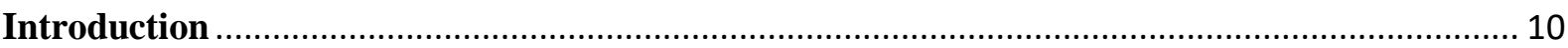

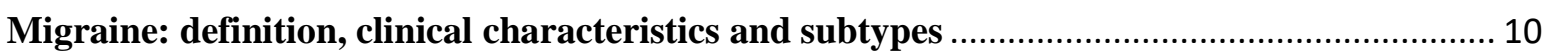

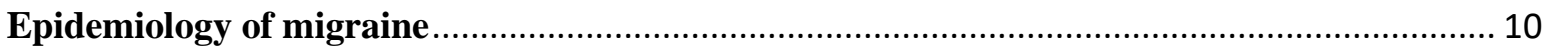

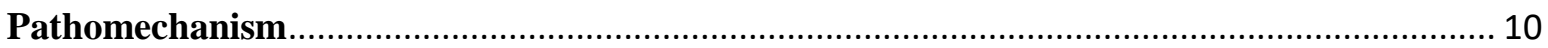

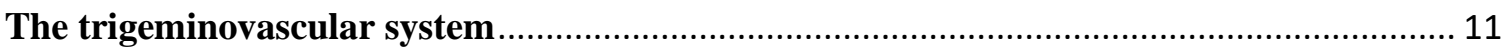

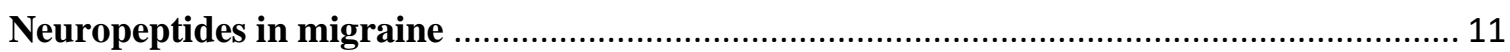

Central sensitization and altered cortical excitability ........................................................ 12

Structural and functional alteration of the brain in migraine ............................................... 13

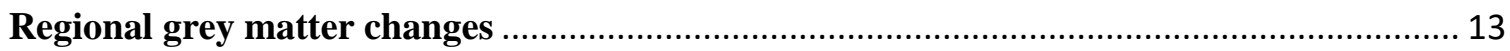

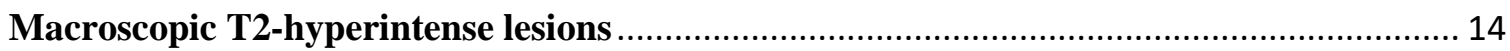

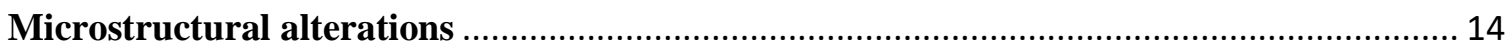

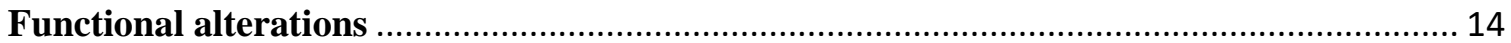

Directions for imaging markers of migraine: methodological advances and relationship to

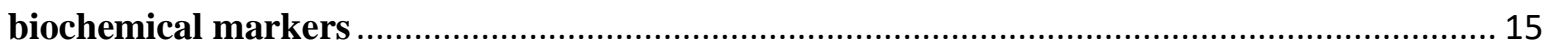

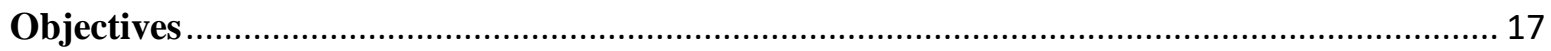

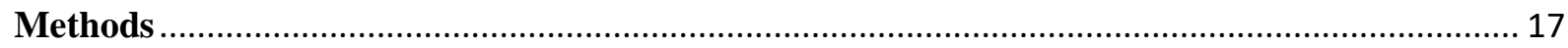

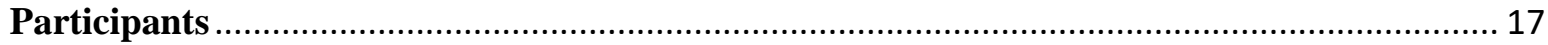

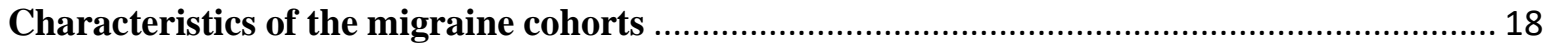

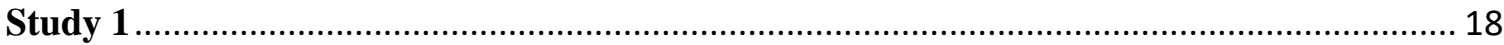

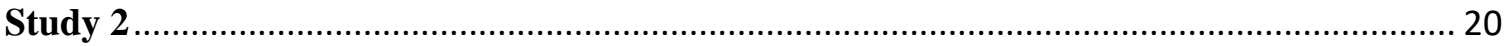

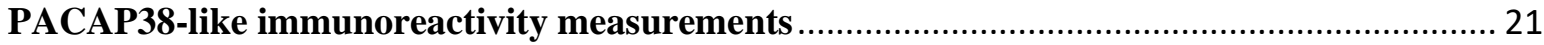

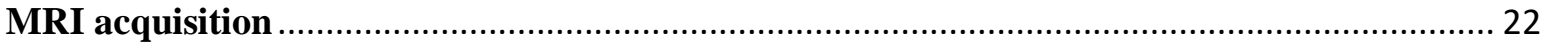

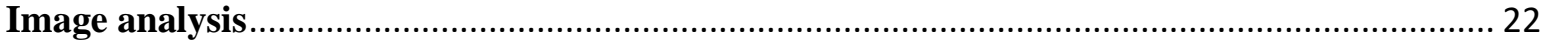

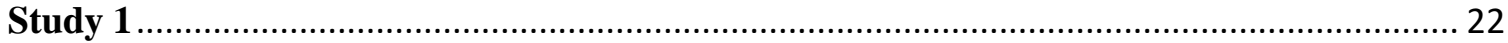

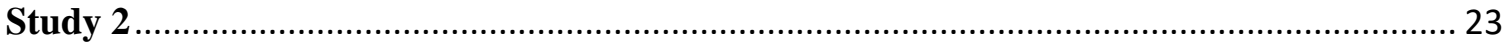

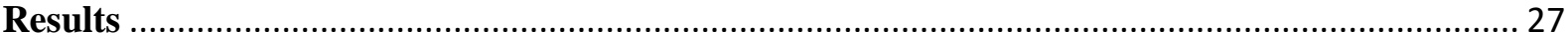

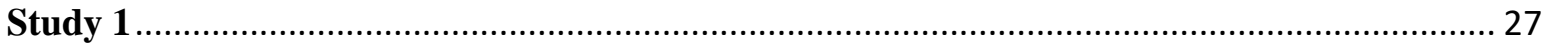




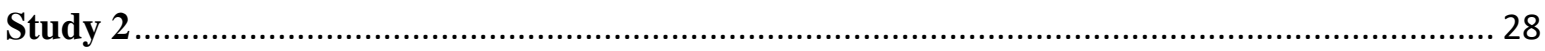

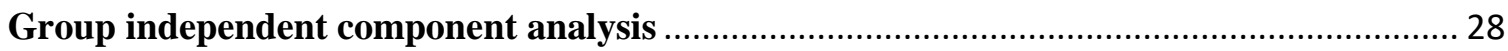

Temporal characteristics of connectivity in the salience network ......................................... 30

Causal interactions of the salience network and other large-scale networks........................ 31

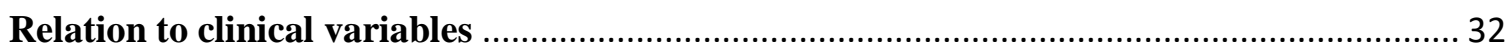

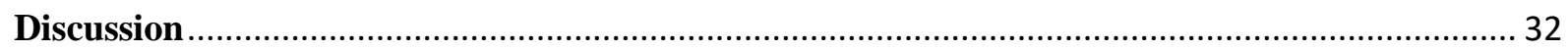

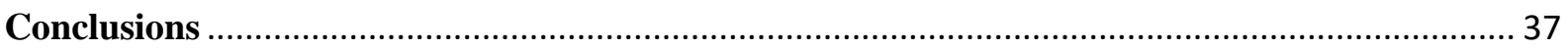

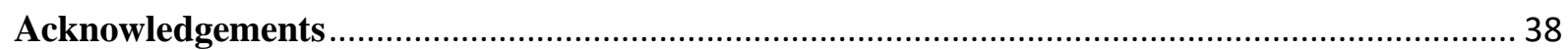

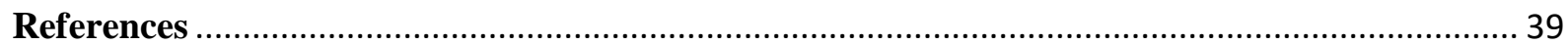




\section{Original publications related to the thesis}

I. Dániel Veréb, Nikoletta Szabó, Bernadett Tuka, János Tajti, András Király, Péter Faragó, Krisztián Kocsis, Eszter Tóth, Bálint Kincses, Teréz Bagoly, Zsuzsanna Helyes, László Vécsei, Zsigmond Tamás Kincses: Correlation of neurochemical and imaging markers in migraine: PACAP38 and DTI measures. Neurology, 2018 Sep 18; 91(12):e1166-e1174. doi: 10.1212/WNL.0000000000006201

\section{IF: 8.77}

II. Dániel Veréb, Nikoletta Szabó, Bernadett Tuka, János Tajti, András Király, Péter Faragó, Krisztián Kocsis, Eszter Tóth, Bence Bozsik, Bálint Kincses, László Vécsei, Zsigmond Tamás Kincses: Temporal instability of salience network activity in migraine with aura. PAIN, April 2020 - Volume 161 - Issue 4 - p 856-864. doi: 10.1097/j.pain.0000000000001770

\section{IF: $\mathbf{5 . 4 8 3}$}

\section{Original publications not directly related to the thesis}

I. Zsigmond Tamás Kincses, Eszter Tóth, Nóra Bankó, Dániel Veréb, Nikoletta Szabó, Gergő Csete, Péter Faragó, András Király, Krisztina Bencsik, László Vécsei: Grey matter atrophy in patients suffering from multiple sclerosis. Clinical Neuroscience, 2014, 67 (9-10), 293-300

\section{IF: 0.337}

II. Nikoletta Szabó, Péter Faragó, András Király, Dániel Veréb, Gergő Csete, Eszter Tóth, Krisztián Kocsis, Bálint Kincses, Bernadett Tuka, Árpád Párdutz, Délia Szok, János Tajti, László Vécsei, Zsigmond Tamás Kincses: Evidence for plastic processes in migraine with aura: a diffusion weighted MRI study. Frontiers in Neuroanatomy, 2018 Jan 17;11:138. doi: 10.3389/fnana.2017.00138

\section{IF: 3.292}

III. Eszter Tóth, Péter Faragó, András Király, Nikoletta Szabó, Dániel Veréb, Krisztián Kocsis, Bálint Kincses, Dániel Sandi, Krisztina Bencsik, László Vécsei, Zsigmond Tamás Kincses: The contribution of various MRI parameters to clinical and cognitive disability in multiple sclerosis. Frontiers in Neurology, 2019 Jan 23; 9:1172. doi: 10.3389/fneur.2018.01172 


\section{IF: 2.889}

IV. Bálint Kincses, Benjámin János Hérák, Nikoletta Szabó, Bence Bozsik, Péter Faragó, András Király, Dániel Veréb, Eszter Tóth, Krisztián Kocsis, Krisztina Bencsik, László Vécsei, Zsigmond Tamás Kincses: Gray matter atrophy to explain subclinical oculomotor deficit in multiple sclerosis. Frontiers in Neurology, 04 June 2019. doi: https://doi.org/10.3389/fneur.2019.00589

\section{IF: 2.889}

V. Péter Faragó, Eszter Tóth, Krisztián Kocsis, Bálint Kincses, Dániel Veréb, András Király, Bence Bozsik, János Tajti, Árpád Párdutz, Délia Szok, László Vécsei, Nikoletta Szabó, Zsigmond Tamás Kincses: Altered resting state functional activity and microstructure of the white matter in migraine with aura. Frontiers in Neurology, 01 October 2019 doi: https://doi.org/10.3389/fneur.2019.01039

\section{IF: 2.889}

VI. Bálint Kincses, Tamás Spisák, Péter Faragó, András Király, Nikoletta Szabó, Dániel Veréb, Krisztián Kocsis, Bence Bozsik, Eszter Tóth, László Vécsei, Zsigmond Tamás Kincses: Brain MRI Diffusion Encoding Direction Number Affects Tract-Based Spatial Statistics Results in Multiple Sclerosis. Journal of Neuroimaging, 2020;00:1-11.DOI: 10.1111/jon. 12705

\section{IF: 2.321}

\section{Review publications}

I. Zsigmond Tamás Kincses, András Király, Dániel Veréb, László Vécsei: Structural magnetic resonance imaging markers of Alzheimer's disease and its retranslation to rodent models. Journal of Alzheimer's Disease, 2015; Doi: 10.3233/JAD-143195

\section{IF: 3.909}

II. Zsigmond Tamás Kincses, Dániel Veréb, Péter Faragó, Eszter Tóth, Krisztián Kocsis, Bálint Kincses, András Király, Bence Bozsik, Árpád Párdutz, Délia Szok, János Tajti, László Vécsei, Bernadett Tuka, Nikoletta Szabó: Are migraine with and without aura really different entities? Frontiers in Neurology, 2019; Doi: 10.3389/fneur.2019.00982

\section{IF: 2.889}


Scientometry:

Total impact factor: 35.6

Citations: 40

H-index: 4 


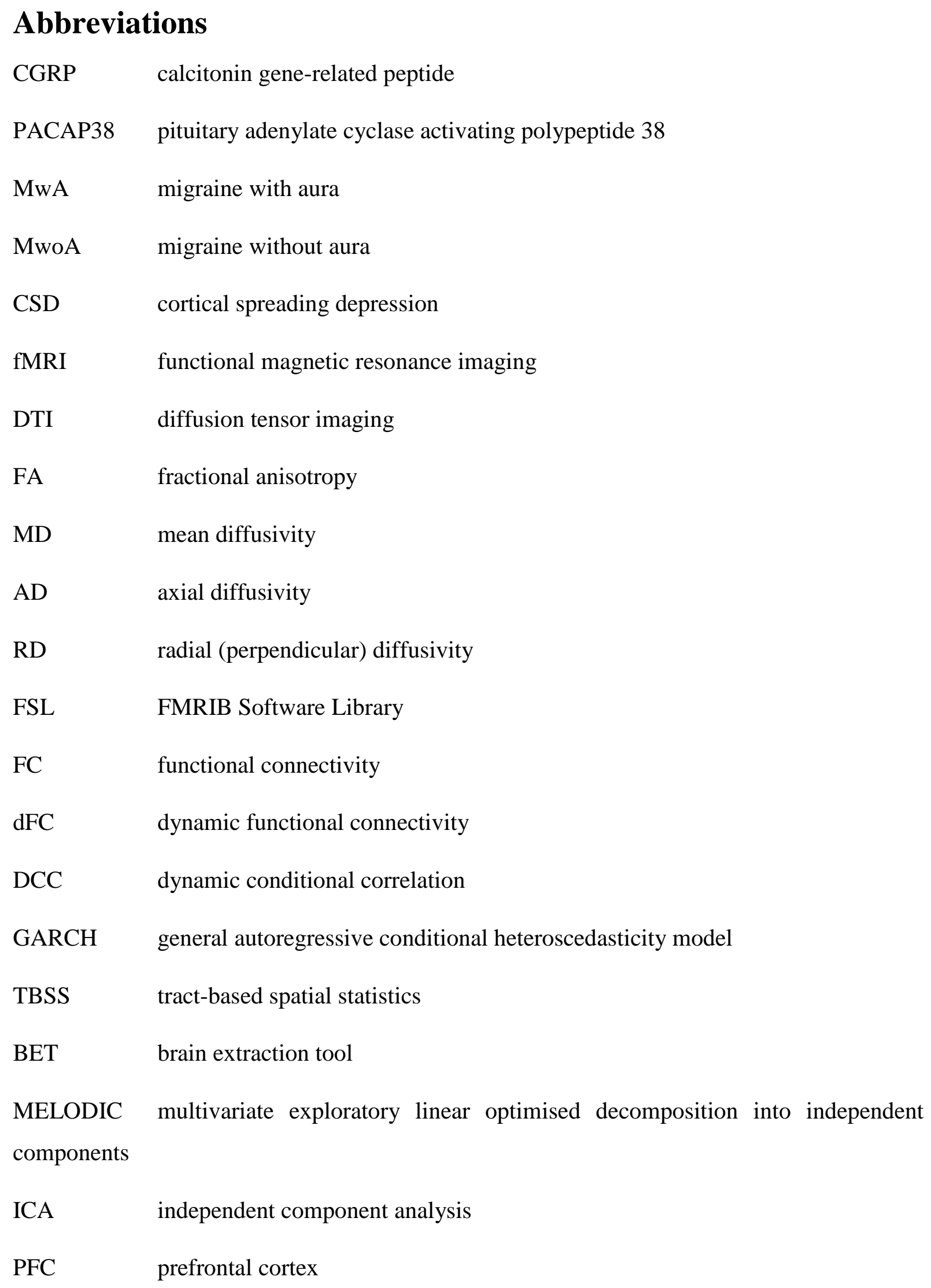


AI

anterior insula

$\mathrm{ACC} \quad$ anterior cingular cortex 


\section{Summary}

\section{Introduction}

Migraine is a common primary headache disorder, yet the exact cause and processes underlying the disease are not fully clear. Neuroimaging markers have proven useful in tracking the disease course and acquiring information about the pathomechanism. However, questions remain about the background of identified alterations and their connection to other biomarkers.

\section{Objectives}

In this work, we aim to place established alterations of white matter microstructure in the context of neurochemical alterations. Furthermore, we set out to identify new potential functional markers for migraine employing temporal features of functional connectivity that potentially complement current approaches.

\section{Methods}

In the first study, we use tract-based spatial statistics to investigate how microstructural alterations of the white matter relate to interictal serum levels of PACAP38, a neuropeptide implicated in migraine pathogenesis, in a cohort of 26 migraine patients. In the second study, we investigate how temporal features of functional connectivity in the salience network differ between healthy controls $(n=32)$, migraine with $(n=20)$ and migraine without aura patients $(n=37)$, using dynamic conditional correlation. We then proceed to describe the effects of connectivity variability on large-scale network interactions using spectral Granger's causality.

\section{Results}

Serum levels of PACAP38 correlated with mean, axial and radial diffusivity in Study 1 ( $p<0.018, p<0.043, p<0.042$, respectively). These findings were located mainly in the left optic radiation and the left posterior corpus callosum, reaching into the parietal and temporal white matter tracts. When we included disease duration in the regression model, the spatial pattern of findings relocated to the left thalamus (mean and axial diffusivity: $\mathrm{p}<0.01$ ). In Study 2, the temporal variance of correlation was higher in the aura group compared to migraine without aura and healthy controls between the right anterior insula and dorsal anterior cingulate cortex ( $\mathrm{p}<0.011$ and $\mathrm{p}<0.026)$ and also higher in the aura group compared to healthy controls between the left prefrontal cortex and dorsal anterior cingulate cortex $(\mathrm{p}<0.021)$. The sum of causality 
from the salience to the dorsal attention network in the 0.01-0.05 $\mathrm{Hz}$ range was lower in migraine with aura compared to migraine without aura and healthy controls $(p<0.032$ in both cases). In migraine without aura, the variance of right and left prefrontal cortex connectivity and right anterior insula - right prefrontal cortex connectivity diminished with increasing attack frequency $(\mathrm{R}=-0.516, \mathrm{p}<0.003$ and $\mathrm{R}=-0.456, \mathrm{p}<0.012)$. Causal interaction power in the 0.01 $0.05 \mathrm{~Hz}$ range between the default mode and dorsal attention networks diminished with longer disease duration in the migraine with aura group $(\mathrm{R}=-0.525, \mathrm{p}<0.036)$. We also found a relationship between right prefrontal cortex - dorsal anterior cingulate cortex connectivity variance and salience-default mode network causality in the migraine with aura group $(\mathrm{R}=$ $0.564, \mathrm{p}<0.045)$.

\section{Conclusions}

We identified a link between microstructural characteristics of the white matter and serum levels of PACAP38 in the interictal term in migraine patients, which connects neuroimaging and neurochemical markers of migraine, further emphasizing the values of both markers. The second study showed that temporal features of connectivity might be an alternative to current functional connectivity markers in migraine and presents evidence that pathophysiological differences between the main subtypes might manifest in altered brain network function that can be used to objectively differentiate between them. 


\section{Introduction}

\section{Migraine: definition, clinical characteristics and subtypes}

Migraine is a primary headache disorder classically manifesting in attacks of unilateral, throbbing headache with accompanying vegetative and sometimes focal neurological symptoms [1]. In the main subtype, migraine without aura, the attacks usually last 4-72 hours and are moderate to severe in headache intensity, exacerbated by physical activity. Other symptoms can include nausea, vomiting and sensitivity to various environmental effects (most frequently light and noise [2]). A prodromal stage may precede the attacks, often associated with fatigue, difficulty concentrating, neck stiffness or blurred vision. These symptoms may also appear during the 48 hours following the headache and are then termed as the postdromal stage. The other subtype, migraine with aura, is mainly characterised by additional transient, focal neurological symptoms preceding or accompanying the attacks called aura [3]. Aura symptoms are most commonly visual in nature and include blurry vision, scotomas or photopsia. Other types of aura may include somatosensory (numbness, paraesthesia) or motor symptoms (transitory dysphasia).

\section{Epidemiology of migraine}

Migraine is among the most frequent disabling neurological disorders, estimated to affect around 1 billion people worldwide and 80 million people in Western Europe alone [4], and might still be under-recognised [5]. In Hungary, a population-based epidemiology study reported a $7.6 \%$ one-year prevalence for migraine without aura and $2 \%$ for migraine with aura [6]. The disorder predominantly affects women: the age-standardised global prevalence was recently estimated as $18.9 \%$ for females and $9.8 \%$ for males [4].

\section{Pathomechanism}

Migraine is a disease with a heterogeneous, complex background. Although classically regarded as a disorder of vascular origin, the consensus view has since evolved to regard it as a disease of the brain [7] that involves widespread and intricate alterations of sensory function [8] and brain energy metabolism [9]. 


\section{The trigeminovascular system}

A key role of the activation and sensitization of the trigeminovascular system has been described in the development of migraine attacks [10]. As part of the trigeminal nerve, perivascular afferents from dural vessels transmit nociceptive information to the caudal trigeminal nucleus. In migraine, a local sterile inflammation develops that coincides with the release of vasoactive peptides from trigeminal afferents (e.g. CGRP, substance P, PACAP38). These peptides induce the dilation of meningeal vessels, plasma extravasation and the degranulation of mast cells in the dura which contributes to a persistent sensitization of meningeal nociceptors $[11,12]$.

\section{Neuropeptides in migraine}

Neuropeptides have been an important research target in migraine, and present opportunities for therapeutic intervention [13]. The most important ones include calcitonin gene-related peptide (CGRP), vasoactive intestinal peptide (VIP), substance P (SP), neuropeptide Y (NPY) and pituitary adenylate-cyclase-activating polypeptide 38 (PACAP38). A pivotal role of CGRP in the pathomechanism of migraine has been established in the 90s [14]. It shows widespread expression in several structures implicated in migraine, such as the trigeminal ganglion and nucleus caudalis (pars caudalis of the spinal trigeminal nucleus) or the locus coeruleus $[15,16]$ (the latter suggested to be a "migraine generator" region beside the periaqueductal grey matter and the raphe nuclei [17]). In migraine patients, the expression of CGRP-receptors is upregulated in the trigeminal nucleus caudalis [18]. During the neuronal activation of the trigeminal ganglion, release of CGRP stimulates satellite glial cells, which results in the release of proinflammatory cytokines, further modulating neuronal function [19]. Apart from this, CGRP functions as a potent vasodilator in the meningeal vasculature [20], and contributes to neurogenic inflammation and mast cell degranulation in the dura by way of the CGRPimmunoreactive branches of the trigeminal ganglion [21, 22]. Furthermore, a direct relationship exists between plasma levels of CGRP and migraine attacks: CGRP-levels increase during nitrogen monoxide-induced migraine attacks and return to baseline after the headache ceases or is treated with triptans [23, 24]. Given intravenously, CGRP also triggers migraine-like headache in migraine patients [25]. Recent advancements in prophylactic migraine therapy also include CGRP-pathway targeting monoclonal antibodies erenumab (which targets the CGRPreceptors), fremanezumab and galcanezumab (which target the CGRP molecule) [26]. 
Another peptide of growing importance in the migraine literature is PACAP38, a molecule which exhibits high structural homology with VIP [27]. PACAP38 is ubiquitous in the body, appearing in different tissues and subserving a range of biological functions, such as vasodilation, neurogenic inflammation and sensitization [28-31]. PACAP38 and its receptors have been detected in several migraine-related regions of the central nervous system: the trigeminal ganglion [32], trigeminal nucleus caudalis [16], locus coeruleus [33], periaqueductal grey matter [15], raphe nuclei [34], thalamus [35] among others. Binding sites include regions of the cortex, thalamus, hypothalamus and brainstem [36], the trigeminal ganglion [37], human mast cells [38] and meningeal arteries [39]. The peptide was implicated in migraine as a biomarker when it was shown that PACAP38-levels in the plasma elevate during migraine attacks similarly to CGRP, and return to a baseline level lower than that of healthy controls in the interictal term [40]. PACAP38 seems to have migraine-inducing properties as well: giving the peptide intravenously triggers migraine-like headache attacks with the dilation of extracranial arteries [41, 42], which evoke changes in brain function similar to alterations during a typical migraine attack [43]. In animal models, infusion of PACAP38 also elicits photophobia [44]. Studies have been trying to exploit the seemingly specific role of PACAP38 in migraine pathomechanism to devise new therapeutic targets, with the blockade of the PAC1 receptor being the most promising method [45].

\section{Central sensitization and altered cortical excitability}

In a large proportion of migraine patients, central sensitization of higher order neurons also develops [46, 47]. This can lead to cutaneous allodynia and might contribute to other sensory symptoms of migraine. Central projections of meningeal trigeminal afferents proceed to the caudal trigeminal nucleus and are relayed to various areas of the brain including brainstem nuclei (periaqueductal grey matter, locus coeruleus) and the hypothalamus and thalamus [46]. From these structures, further ascending projections eventually reach cortical regions including areas related to sensation (somatosensory, visual, auditory, insular cortices) and higher order areas (parietal association cortex, prefrontal cortices). These connections might contribute to sensory symptoms of migraine apart from the headache.

Another important mechanism that is thought to underlie the aura phenomenon is cortical spreading depression (CSD, [48, 49]), a slow wave of depolarisation that moves across the cortex with a velocity of $3-5 \mathrm{~mm} / \mathrm{min}$ [49]. The depolarization is followed by a long-lasting 
depression of neurons and is accompanied by a perturbation of brain metabolism and blood circulation.

Baseline brain metabolism is also affected in migraine, which presents with decreased mitochondrial energy storage [9]. As a sign of hindered oxidative glycolysis, increased lactate metabolite levels have been reported in different migraine groups [50, 51], whereas $\mathrm{N}$-acetylaspartate (NAA) levels have been shown to decrease both ictally and interictally [52]. In migraine without aura, a decreased NAA/creatine ratio was associated with greater attack frequency and disease duration in the occipital lobe [53].

\section{Structural and functional alteration of the brain in migraine}

Disease processes leave their mark on the brain in migraine: a plethora of neuroimaging studies conducted in recent years have found widespread alteration in the structure and function of migraine patients' brain compared to healthy controls [54-57]. Structural alterations can mostly be categorised into three groups: regional grey matter volume changes, macroscopic T2hyperintense white matter lesions, and microstructural alterations of the white matter; it is also important to note that these alterations can be interconnected $[58,59]$.

\section{Regional grey matter changes}

Regarding cortical thickness, migraine patients exhibit loss of grey matter in several brain regions related to pain processing, including the prefrontal cortex (PFC), insula and periaqueductal grey matter [60-63]. These changes were tied to clinical characteristics, with increased headache frequency and duration being associated with progressive grey matter loss in these areas [60]. However, it is still debated whether these changes are pathognomic in migraine and reflect the underlying pathogenesis or they are a more general and reversible consequence of chronic exposure to an increased allostatic load posed by the disease [64, 65]. Apart from symptomatology-related changes, it is difficult to pinpoint brain areas involved in the pathogenesis; studies report conflicting results, which might stem from inconsistent methodology or the heterogeneity of examined migraine cohorts (e.g. a current study by Schwedt et al. was able to subdivide even a fairly homogeneous group of migraine patients into two subgroups based on structural MRI measurements [66]). Several studies also reported no significant changes of grey matter density in migraine compared to healthy controls [67, 68]. To tackle heterogeneity in migraine cohorts, a recent study employed a coordinate-based 
network mapping approach, which highlighted the role of area V3 (formerly implicated in the development of CSD [69], and shown to differ from healthy controls in structure by several previous studies (e.g. [70])) as a specific migraine-related site [71].

\section{Macroscopic T2-hyperintense lesions}

Migraine (both with and without aura) reportedly poses an increased risk for subclinical infarcts in the posterior circulation and deep white matter lesions (WML), macroscopically visible as hyperintense regions on T2-weighted MRI scans [72, 73]. Etiologically, WMLs were proposed to result from temporary disruption of the blood-brain-barrier by a matrix-metalloprotease-9related mechanism [74]. The clinical importance of these lesions is not entirely clear, though some studies report an association between headache frequency and the risk for developing WMLs [73].

\section{Microstructural alterations}

Apart from macroscopic white matter lesions, an increasing number of studies (mostly employing diffusion tensor imaging [75]) report microstructural changes in the white matter of migraine patients $[54,55,76]$. These changes usually affect visual and pain-related pathways, and though their exact origin remains unclear, microstructural alterations were hypothesised to be a sign of maladaptive plasticity or degeneration in response to the continuous allostatic burden migraineurs experience in the form of headache attacks [54, 77-79]. A study suggested that disturbances in the posterior circulation (an increased right-to-left shunt) correspond to altered microstructure in the right corona radiata and the body of the corpus callosum [80]. Furthermore, there are microstructural differences in the white matter even between migraine subgroups, exhibiting a dissimilar pattern in migraine with and without aura, which complicates the underlying mechanism as well [55]. Though their origins remain subject to debate, white matter microstructural alterations show promise as biomarkers in chronic pain conditions including migraine [81].

\section{Functional alterations}

Functional MRI studies investigating migraine have found alterations during the headache attack and in the interictal period as well [57].

During the ictal phase, resting state connectivity within and between large-scale networks is different from that of healthy controls. Patients with aura exhibit increased functional 
connectivity (FC) of sensory areas, especially the visual and somatosensory cortices [82]. Similar changes appear during artificially induced migraine attacks, where migraine patients show altered connectivity in the salience, default mode and sensorimotor networks after intravenous infusion of PACAP38 [43]. Higher order systems are also affected: a study found reduced FC between the executive and dorsal attention networks that correlated with headache attack frequency in migraine without aura patients [83], which falls in line with studies showing cognitive disturbances during the ictal phase [84]. These baseline, resting state changes carry over to task performance as well $[85,86]$ and are consistent with earlier reports of altered cortical excitability in migraineurs both during the aura phase [87] and in the interictal term [88].

Studies investigating connectivity in the interictal phase report more conflicting results. Widespread connectivity changes were found in limbic [89], sensory and motor brain regions [90] and in the executive [91] and default mode networks [92]. Several studies also report differences between migraine with and without aura patients, mainly in the visual and executive networks [56, 93, 94], while a study found no differences in functional connectivity between migraine patients and healthy controls [95]. Conflicting results in these studies might be attributed to methodological differences in the estimation of functional connectivity and some studies used mixed groups, disregarding possible further differences between migraine subtypes.

\section{Directions for imaging markers of migraine: methodological advances and relationship to biochemical markers}

One way to disentangle confounding results in imaging markers is to examine them in a broader context, e.g. in relation to neurochemical alterations in the patients. This could also lead to novel insights about the pathomechanism, and how neurochemical and structural aspects of migraine interact. PACAP38 as a migraine-related neuropeptide is a viable candidate for this because its serum levels approximately represent intracerebral PACAP38 metabolism: the peptide is able to traverse the blood-brain-barrier via a saturable transport mechanism [96] (although some studies question its direct central effects in the case of migraine [97]). Since PACAP38 acts as a functional molecule in several migraine-related systems [98, 99], it is possible that changes in the peptide's metabolism in migraine might be expressed as or co- 
occur with microstructural alterations of the white matter. These alterations were previously suggested to be a sign of plastic remodelling or degenerative changes [54]. Since PACAP38 alters levels of markers that indicate glial or neuronal damage (migraine patients exhibited altered levels of S100B in the plasma after an infusion of PACAP38 [100]), and levels of these markers reportedly increases during migraine attacks in migraine without aura [101], it is possible that PACAP38 induces degenerative microstructural changes in the white matter of migraine patients that might be detectable with diffusion tensor imaging. Alternatively, neurotrophic and neuroprotective effects of PACAP38 have also been described [102, 103], which might engender plastic changes in the brain that diffusion-weighted MRI sequences are able to detect.

Other possible directions have been opened up by recent advances in the methodology of functional connectivity estimation [104]. A growing number of studies assert that functional connectivity between brain regions changes over time, on several time scales $[105,106]$. These findings gained even more traction since time-varying connectivity changes were detected in different neuropsychiatric conditions, e.g. in schizophrenia [107], autism [108], Alzheimer's disease [109], Parkinson's disease [110] and multiple sclerosis [111], among others [112]. In migraine, different thalamocortical network dynamics were found compared to controls [113], and based on dynamic functional connectivity, a study was able to determine whether a participant suffered from a migraine headache with a sensitivity of 0.7 and a specificity of 0.76 [114]. These findings correspond to a recent model of increased sensory gain in brain networks affected by migraine, which would suggest more flexible intra- and internetwork connections that come with an altered response or adaptation to extrinsic stimuli [8]. Altered levels of excitatory and inhibitory neurotransmitters were described in migraine patients (e.g. glutamate and GABA, see [115] for a review), which, accompanied by metabolic disturbances, could manifest as imbalance in network function. Temporal dynamics of intrinsic and betweennetwork connectivity might be suitable markers of this imbalance, which is possibly more emphasised in migraine patients with aura, due to a more frequent or emphasised occurrence of disease features like cortical hyperexcitability or cortical spreading depression [116]. In a model of widespread sensory disturbance, the salience network is a network of interest because it putatively plays a role in the transition between the default mode and task-positive networks [117-119], sorting through extrinsic stimuli and distinguishing between those unimportant and 
important to the person, therefore allocating more attentional resources to salient stimuli. However, hyperexcitable brain regions respond disproportionately to environmental stimuli, which makes it harder to determine whether a stimulus is salient or not. Migraine patients, especially those experiencing aura symptoms, exhibit altered excitability in numerous brain regions (mostly the visual and somatosensory cortices [87, 88]) and, accordingly, their performance is inferior to healthy controls in tasks where they have to distinguish between target stimuli and a noisy background (e.g. motion detection tasks [120]). The salience network could be a promising target to capture a network level expression of altered excitability. Regions belonging to this network exhibit temporal changes of connectivity that scales with disease duration in temporal lobe epilepsy, another disorder where cortical hyperexcitability occurs and which shares pathophysiological features with migraine [121-124]. Therefore, we hypothesised that migraine patients, especially those with aura symptoms, also show altered temporal features of connectivity in the salience network even in the interictal term, which possibly affect connectivity between large-scale brain networks and appear as a function of the patients' clinical condition.

\section{Objectives}

Our aim was to identify novel structural and functional markers of migraine with and without aura and to explore the relationship between peripheral levels of PACAP38 and central alterations. In Study 1, our hypothesis was that microstructural properties of migraine-related white matter tracts are associated with altered interictal levels of PACAP38 in the serum. In Study 2, we looked for alterations of intrinsic salience network connectivity in migraine with and without aura patients and then examined the relationship between intrinsic salience network connectivity and large-scale network interaction, as a function of clinical status.

\section{Methods}

\section{Participants}

For the first study, we recruited 26 migraine patients ( 8 with aura). The second study involved 20 migraineurs with and 37 migraineurs without aura in addition to 32 age and sex-matched controls. All migraine patients were treated as outpatients at the Headache Outpatient Clinic, at the Department of Neurology, University of Szeged. Migraine was diagnosed on the basis of the International Headache Society criteria [1] by expert neurologists with at least 10 years of 
experience. All participants provided their written consent as required by the Helsinki Declaration, and both studies were approved by the local ethics committee (reference number 87/2009).

\section{Characteristics of the migraine cohorts}

\section{Study 1}

The clinical and demographic characteristics of the patients are summarised in Table 1. Exclusion criteria entailed a Hamilton Depression Scale score of $>8$, abuse of alcohol or illicit drug use and any neuropsychiatric disorders apart from migraine. 8 patients received prophylactic treatment for migraine (2 topiramate, 6 iprazochrome). 
Table 1

Demographic and clinical data of Study 1 participants. Abbreviations: $y=y e a r$,

$\mathrm{SD}=$ standard deviation, $\mathrm{VAS}=$ visual analog scale, $\mathrm{R}=$ right, $\mathrm{L}=$ left, $\mathrm{A}=$ alternating,

MwoA=migraine without aura, MwA=migraine with aura, $\mathrm{F}=$ female, $\mathrm{M}=$ male.

\begin{tabular}{|c|c|c|c|c|c|c|c|c|}
\hline Patient & Age, $y$ & Sex & Type & $\begin{array}{l}\text { Disease } \\
\text { duratio } \\
\mathbf{n}, \mathbf{y}\end{array}$ & $\begin{array}{l}\text { Attack frequency, } \\
\text { attacks/y }\end{array}$ & $\begin{array}{l}\text { Allody } \\
\text { nia } \\
\text { score }\end{array}$ & VAS & $\begin{array}{l}\text { Heada } \\
\text { che } \\
\text { side }\end{array}$ \\
\hline 1 & 33 & $\mathrm{~F}$ & MwoA & 15 & 36 & 1 & 7 & $\mathbf{A}$ \\
\hline 2 & 34 & $\mathrm{~F}$ & MwoA & 3 & 52 & 8 & 8 & $\mathbf{R}$ \\
\hline 3 & 54 & $\mathrm{~F}$ & MwoA & 20 & 12 & 0 & 10 & $\mathbf{R}$ \\
\hline 4 & 30 & $\mathrm{~F}$ & MwA & 16 & 18 & 2 & 6 & $\mathbf{L}$ \\
\hline 5 & 29 & $\mathrm{~F}$ & MwoA & 18 & 36 & 2 & 8 & $\mathbf{L}$ \\
\hline 6 & 38 & $\mathrm{~F}$ & MwoA & 30 & 60 & 6 & 9 & $\mathbf{A}$ \\
\hline 7 & 53 & $\mathrm{~F}$ & MwoA & 24 & 12 & 10 & 5 & $\mathbf{L}$ \\
\hline 8 & 23 & $\mathrm{~F}$ & MwA & 8 & 72 & 2 & 7 & $\mathbf{R}$ \\
\hline 9 & 21 & $\mathrm{~F}$ & MwoA & 1 & 12 & 0 & 10 & $\mathbf{L}$ \\
\hline 10 & 27 & $\mathrm{~F}$ & MwoA & 3 & 52 & 9 & 7 & $\mathbf{L}$ \\
\hline 11 & 38 & $\mathrm{~F}$ & MwoA & 13 & 120 & 0 & 9 & $\mathbf{A}$ \\
\hline 12 & 24 & M & MwA & 7 & 1 & 0 & 7 & $\mathbf{R}$ \\
\hline 13 & 44 & $\mathrm{~F}$ & MwoA & 32 & 24 & 2 & 9 & $\mathbf{A}$ \\
\hline 14 & 37 & $\mathrm{~F}$ & MwA & 9 & 3 & 2 & 7 & $\mathbf{A}$ \\
\hline 15 & 37 & $\mathrm{~F}$ & MwoA & 27 & 36 & 0 & 9 & $\mathbf{A}$ \\
\hline 16 & 33 & $\mathrm{~F}$ & MwoA & 15 & 48 & 0 & 9 & $\mathbf{A}$ \\
\hline 17 & 28 & $\mathrm{~F}$ & MwoA & 5 & 120 & 4 & 7 & $\mathbf{A}$ \\
\hline 18 & 46 & $\mathrm{~F}$ & MwoA & 31 & 30 & 0 & 8 & $\mathbf{A}$ \\
\hline 19 & 29 & $\mathrm{~F}$ & MwA & 10 & 6 & 2 & 8 & $\mathbf{A}$ \\
\hline 20 & 35 & $\mathrm{~F}$ & MwA & 18 & 53 & 4 & 8 & $\mathbf{L}$ \\
\hline 21 & 28 & $\mathrm{~F}$ & MwoA & 4 & 60 & 3 & 9 & $\mathbf{A}$ \\
\hline 22 & 25 & $\mathrm{~F}$ & MwoA & 7 & 36 & 8 & 8 & $\mathbf{L}$ \\
\hline 23 & 47 & $\mathrm{~F}$ & MwoA & 11 & 182 & 6 & 8 & $\mathbf{R}$ \\
\hline 24 & 38 & $\mathrm{~F}$ & MwoA & 12 & 30 & 2 & 10 & $\mathbf{A}$ \\
\hline 25 & 24 & M & MwA & 11 & 8 & 0 & 6 & $\mathbf{A}$ \\
\hline 26 & 42 & $\mathrm{~F}$ & MwA & 31 & 36 & 0 & 7 & $\mathbf{R}$ \\
\hline $\begin{array}{l}\text { Mean } \pm \\
\text { SD }\end{array}$ & $34 \pm 9.05$ & & & $14.65 \pm 9.54$ & $44.42 \pm 41.59$ & $\begin{array}{l}\text { Median 2, } \\
\text { mode } 0\end{array}$ & $\begin{array}{l}\text { Median 8, } \\
\text { mode } 7\end{array}$ & \\
\hline
\end{tabular}




\section{Study 2}

Clinical and demographic characteristics of the second study cohort are summarised in Table 2. Similarly to the previous study, participants were screened for depression, psychoactive substance abuse and neuropsychiatric disorders in addition to migraine. Healthy controls did not have any chronic conditions and did not take regular medication apart from oral contraceptives. As this study employed a case-control design, we matched the groups in terms of age, sex distribution and body mass index (one-way analysis of variance for age and body mass index: $p<0.371, p<0.700$ and Fisher exact test for sex: $p<0.744)$. Some patients received interval therapy for migraine (migraine with aura group: 1 iprazochrome, migraine without aura group: 5 iprazochrome, 1 amitriptyline, and 1 topiramate). Aura symptoms were predominantly visual for all participants in the migraine with aura group, and mostly involved photopsia, blurry vision and/or scotomas. Two patients had additional somatosensory symptoms (unilateral numbness). Migraine attacks in the aura group were almost always preceded by aura symptoms, whereas migraineurs without aura never experienced aura symptoms. 


\section{Table 2}

Demographic and clinical data of Study 2 participants. Abbreviations: SD=standard deviation, VAS=visual analog scale.

\begin{tabular}{|l|l|l|l|}
\hline & Healthy controls & $\begin{array}{l}\text { Migraine without } \\
\text { aura }\end{array}$ & Migraine with aura \\
\hline N & 32 & 37 & 20 \\
\hline Age (years, mean \pm SD) & $35.4 \pm 11.3$ & $35.9 \pm 9.1$ & $32.2 \pm 7.8$ \\
\hline Sex (male/female) & $3 / 29$ & $3 / 34$ & $3 / 17$ \\
\hline Disease duration (years, mean \pm SD) & - & $15.4 \pm 11.2$ & $15.4 \pm 8.4$ \\
\hline $\begin{array}{l}\text { Attack frequency (total attacks/year, } \\
\text { mean } \pm \text { SD) }\end{array}$ & - & $54.3 \pm 43.9$ & $28.3 \pm 24.8$ \\
\hline \begin{tabular}{l} 
Attack duration (hours, mean \pm SD) \\
\hline Allodynia score (mean \pm SD)
\end{tabular} & - & $31.4 \pm 26.4$ & $19.9 \pm 18.3$ \\
\hline $\begin{array}{l}\text { Pain intensity during headache (VAS, } \\
\text { mean } \pm \text { SD) }\end{array}$ & - & $3.6 \pm 3.7$ & $1.6 \pm 1.7$ \\
\hline
\end{tabular}

\section{PACAP38-like immunoreactivity measurements}

Regarding Study 1, PACAP38-like immunoreactivity measurements were carried out in collaboration with the Department of Pharmacology and Pharmacotherapy at the Faculty of Medicine of the University of Pécs. Blood samples were drawn before the MRI measurements from the patients' cubital vein. The samples were collected in cooled glass tubes containing 12 mg of EDTA and the protease inhibitor aprotinin (Trasylol 1200 IU; Bayer Pharmaceuticals Corp., West Haven, CT). We kept the tubes containing the samples at $4{ }^{\circ} \mathrm{C}$ before centrifugation and stored them at $-80^{\circ} \mathrm{C}$ afterward. PACAP38-like immunoreactivity (PACAP38-LI) measurements were carried out with a specific and sensitive radioimmunoassay method that we also used in earlier studies [40]. Antibodies used were the PACAP38 antiserum "88111-3" raised in rabbits against synthetic peptides bound to bovine thyroglobulin or bovine serum 
albumin. The tracers were labeled with mono- ${ }^{125}$ I. RIA standards were synthetic peptides in concentrations of 0 to $1000 \mathrm{fmol} / \mathrm{mL}$. We prepared the assay in $1 \mathrm{~mL}$ of $0.05 \mathrm{M}(\mathrm{pH}=7.4)$ phosphate buffer that contained $0.1 \mathrm{M}$ sodium chloride, $0.25 \%(\mathrm{w} / \mathrm{v})$ bovine serum albumin, and $0.05 \%$ (wt/vol) sodium azide. After centrifugation at $2000 \mathrm{rpm}, 4^{\circ} \mathrm{C}, 10$ minutes, samples were precipitated using absolute alcohol and after another centrifugation at $2000 \mathrm{rpm}, 4^{\circ} \mathrm{C}$ for 10 minutes, were dried under nitrogen flow and resuspended in $300 \mu \mathrm{L}$ of assay buffer. Finally, we measured the antiserum $(100 \mathrm{~mL}$, diluted 1:10000), the tracer $(100 \mathrm{~mL}, 5000 \mathrm{cpm} /$ tube $)$ and the standard/unknown samples $(100 \mathrm{~mL})$ with the assay buffer into polypropylene tubes.

\section{MRI acquisition}

For both studies, MRI scans were timed so that patients were attack-free for at least one week before the measurement. The scans were carried out using a 1.5T GE Signa Excite HDxt MR scanner (GE Healthcare, Milwaukee, WI). The acquisition protocol comprised structural scans (3-dimensional fast spoiled gradient echo (3D-FSPGR) images with the following parameters: echo time $=4.1$ milliseconds $[\mathrm{ms}]$; repetition time $=10.276 \mathrm{~ms}$; matrix $: 256 \times 256$; field of view: $25 \times 25 \mathrm{~cm}$; flip angle: $15^{\circ}$; in-plane resolution: $1 \times 1 \mathrm{~mm}$; slice thickness: $1 \mathrm{~mm}$ ), 60 directional diffusion-weighted images with 6 nondiffusion-weighted reference volumes for Study 1 (acquisition parameters: echo time $=94.6 \mathrm{~ms}$; repetition time $=13925 \mathrm{~ms}$; matrix: 96 $\times 96$; field of view: $23 \times 23 \mathrm{~cm}$; flip angle: $90^{\circ}$; in-plane resolution: $2.4 \times 2.4 \mathrm{~mm}$; slice thickness: $2.4 \mathrm{~mm}$; $\mathrm{b}=1000 \mathrm{~s} / \mathrm{mm}^{2}$; number of excitations $=2$; array spatial sensitivity encoding technique factor $=2$ ) and 10 minutes ( 200 volumes) of T2*-weighted BOLD-contrast images for Study 2 (GE EPI sequence, acquisition parameters: echo time $=40 \mathrm{~ms}$, repetition time $=3000 \mathrm{~ms}$, matrix: 64 x 64, flip angle: $90^{\circ}$, FOV: 30 x $30 \mathrm{~cm}$, slice thickness: $6 \mathrm{~mm}$, flip angle: $90^{\circ}$ ). Participants received instructions to close their eyes and stay awake and motionless throughout the scans.

\section{Image analysis}

\section{Study 1}

\section{Preprocessing}

Diffusion-weighted scans were preprocessed using tools of the FMRIB Software Library (FSL, [125]). The images underwent correction for eddy currents and motion artifacts by way of a 12 DOF affine registration to the first nondiffusion-weighted reference scan. Next, a diffusion 
tensor model was fitted in each voxel using FSL's Diffusion Toolbox (FDT). Based on the diffusion tensor model we calculated the fractional anisotropy (FA), mean diffusivity (MD) and diffusivity parallel $(\lambda 1$, axial diffusivity, $\mathrm{AD})$ and perpendicular $((\lambda 2+\lambda 3) / 2$, radial diffusivity, $\mathrm{RD})$ to the main diffusion direction.

\section{Statistical analysis}

To assess the relationship between PACAP38-LI and diffusion parameters along the main white matter tracts, we used the Tract Based Spatial Statistics (TBSS) method included in FSL [126]. TBSS projects participants' FA data to an alignment-invariant representation of main white matter tracts (called the "mean FA skeleton") via an optimised non-linear registration and therefore allows unbiased voxel wise analysis of multi-subject diffusion data. We tested the relationship between PACAP38-LI and diffusion parameters in a general linear model framework, with PACAP38-LI as the independent variable and age and sex as nuisance regressors. Significance testing was performed using FSL's randomise, which implements a nonparametric permutation test with threshold-free cluster enhancement to consider the spatial relationship between neighboring voxels, and controls the family wise error rate to correct for multiple comparisons [127, 128].

\section{Study 2}

\section{Preprocessing}

Functional MRI data was preprocessed using FSL FEAT. After discarding the first 2 volumes to exclude saturation effects, the preprocessing pipeline included motion correction via MCFLIRT [129], the removal of non-brain tissue by BET [130], slice-timing correction, and high pass filtering with a $0.01 \mathrm{~Hz}$ cutoff plus removal of linear trends by Gaussian straight line fitting. Volumes were then normalised to the $4 \mathrm{~mm}$ MNI152 template space using a two-stage boundary-based registration method implemented in FSL. To alleviate the effects of head motion, the data was orthogonalised to the 6 motion parameters estimated by MCFLIRT. Residual data was then standardised to zero mean and unit standard deviation. The three groups did not differ in mean, absolute or relative displacement parameters (Kruskal-Wallis test, $\mathrm{p}<$ 0.425 and $\mathrm{p}<0.953)$. 


\section{Statistical analysis}

\section{Group independent component analysis}

We employed group independent component analysis using FSL MELODIC (v. 3.15, [131]) to obtain group average spatial maps of brain networks. Data dimensionality was estimated automatically by Laplace approximation to the posterior evidence of the model order. We classified independent components (ICs) as signal or noise via visual inspection, on the basis of recently published guidelines [132]. For the estimation of individual network time series, we used a dual regression approach which entails regressing the group-average independent component spatial maps against individual functional scans [133]. We investigated 5 nodes of the salience network: the bilateral insula, dorsal anterior cingulate cortex (dACC), and bilateral anterior prefrontal cortices (r/lPFC). Region of interest (ROI) time series were extracted as the first temporal eigenvariate of time series under the group average salience network node masks. Node ROIs were identified using FSL's cluster tool.

\section{Dynamic conditional correlation}

We characterised the temporal aspect of connectivity between salience network nodes using dynamic conditional correlation (DCC), a novel model-based metric that provides frame wise estimates of correlation and was demonstrated to be more reliable than the widely used sliding window correlation $[134,135]$. DCC is calculated in two stages: first, a general autoregressive conditional heteroscedasticity model $(\operatorname{GARCH}(1,1)[136])$ is fitted for each time series that describes conditional variance at each time point as a function of previous time point residuals and their variance. The parameters of this model can be used to compute standardised residuals, which provide frame wise estimates of correlation between two time series. The method has been evaluated for usage with fMRI data [134] and was shown to be fairly reproducible [135]. To further characterise intrinsic correlation in the salience network, we also calculated the temporal average of correlation values. Since traditional parametric methods are less sensitive when faced with a higher number of variables, differences between groups in terms of the correlation variability and average correlation matrices were assessed using a general linear model approach that employs a nonparametric permutation test for significance testing. This approach is similar to Study 1 except that correction for multiple comparisons was carried out in a "voxel wise" manner without assessing the adjacency of values in the matrices, treating entries as independent from each other [127]. 


\section{Granger's causality}

We used Granger's causality to assess information flow between the salience network and two other major networks in downstream stimulus processing, the default mode and dorsal attention networks [137]. Although prone to limitations [138], Granger's causality is a viable method in fMRI analysis on the basis that certain conditions are met [139]. In this study, we analyzed network time series that do not exhibit significant zero-lag correlation, and regional hemodynamic characteristics are averaged out during network time course estimation. Furthermore, we analyzed group differences, which rather means a difference in causality due to group effects than actual baseline causality. We calculated spectral Granger's causality which estimates the fraction of power at a certain frequency in time series X2 that is supplied by time series X1 [140].

\section{Relation to clinical variables}

Spearman's rank correlation was calculated to assess the relationship between connectivity variance, spectral causal interactions and clinical variables (attack frequency and disease duration). The significance threshold was adjusted according to Bonferroni to correct for multiple hypothesis testing. 


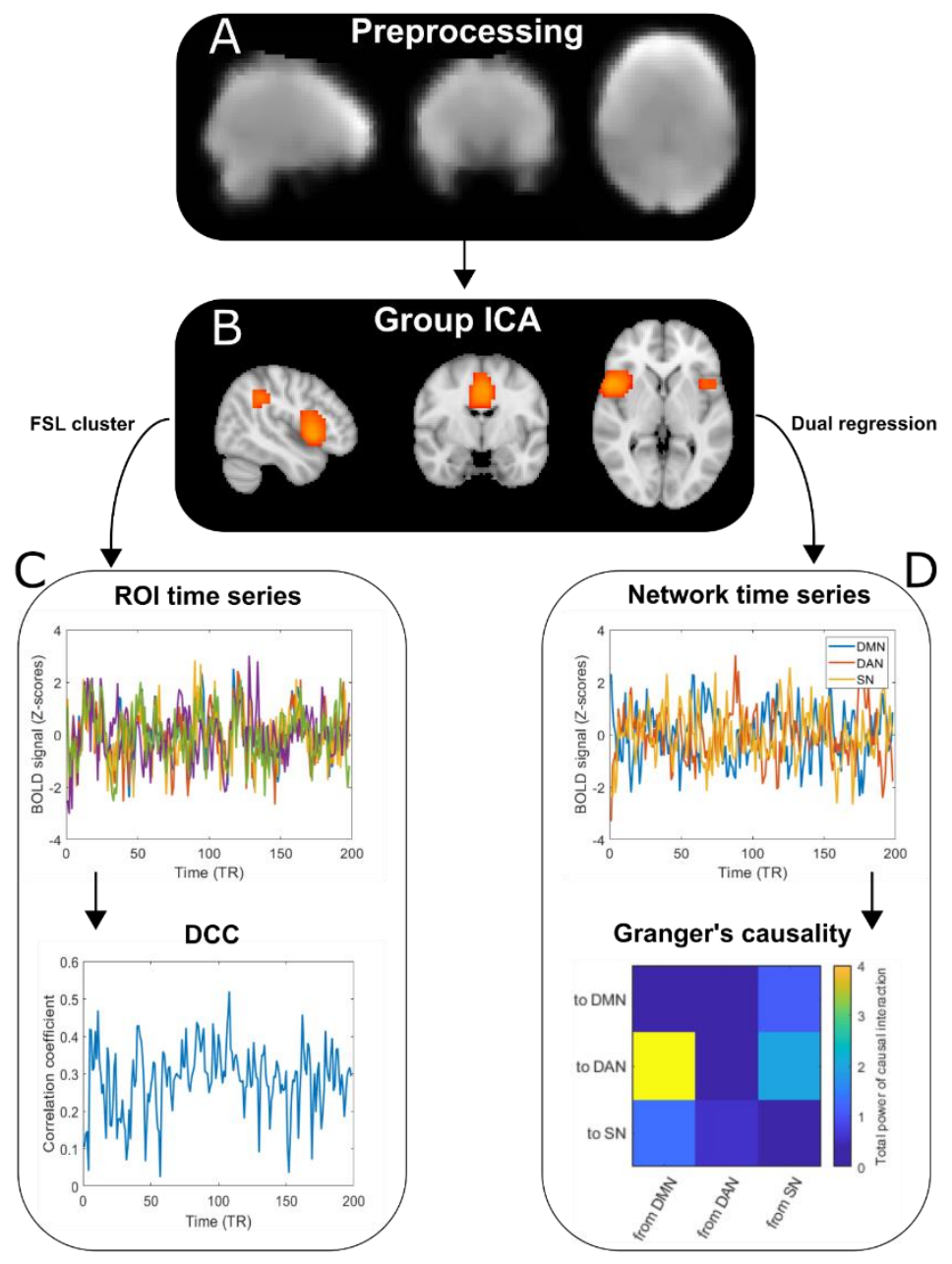

Figure 1: Analysis flow chart for Study 2. (A) Pre-processing for fMRI scans included brain extraction, motion correction, spatial smoothing, nuisance regression and temporal filtering. (B) Resulting volumes were further analysed using temporal concatenation ICA with FSL's MELODIC. (C) Thresholded group average component maps were segmented using FSL's cluster tool to obtain spatial maps of individual salience network nodes. These spatial maps were used to extract time series, from which dynamic conditional correlation was calculated (for details see Methods). Temporal variance and average of dynamic conditional correlation values were compared between groups using randomise. (D) Spectral Granger's causality was calculated between pairs of individual network time series, obtained through dual regression. The sum of causal interactions in the $0.01<0.5 \mathrm{~Hz}$ and $0.05<0.1 \mathrm{~Hz}$ range was compared between groups using randomise. 


\section{Results}

\section{Study 1}

The correlation between interictal PACAP38 and several diffusion parameters was statistically significant (MD: $p<0.018, A D: p<0.043, R D: p<0.042$ ). These findings were located mainly in the left optic radiation and the left posterior corpus callosum, reaching into the parietal and temporal white matter tracts (see Figure 2). When we included disease duration in the regression model, the spatial pattern of findings relocated to the left thalamus (MD and AD: $\mathrm{p}<0.01$ ).
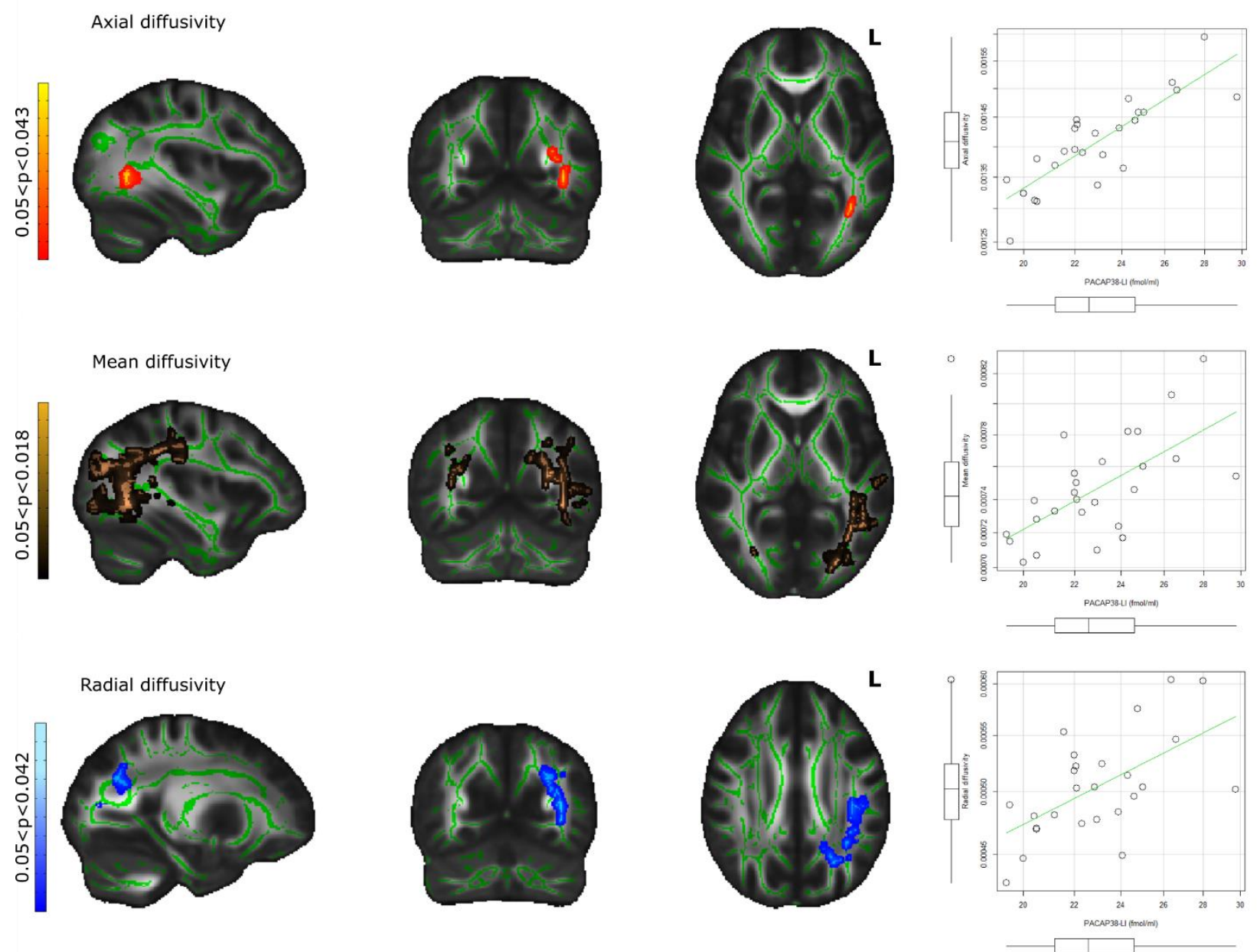

Figure 2: Spatial patterns of correlation between diffusion parameters and PACAP38-LI. The mean FA skeleton is overlaid in green on the mean FA image. Voxels exhibiting significant correlation with PACAP38 are depicted in red-yellow for axial diffusivity (maximum p-value MNI coordinates: $\mathrm{x}=125 \mathrm{y}=69 \mathrm{z}=75$ ), copper for mean diffusivity (maximum $\mathrm{p}$-value MNI coordinates: $\mathrm{x}=125 \mathrm{y}=69 \mathrm{z}=75$ ) and blue-lightblue for radial/perpendicular diffusivity 
(maximum p-value MNI coordinates: $\mathrm{x}=115 \mathrm{y}=64 \mathrm{z}=102$ ). Clusters have been thickened for better visualisation using FSL's tbss_fill tool. The colorbar represents $\mathrm{p}$ values corrected for multiple comparisons. The scatterplots depict PACAP-like immunoreactivity plotted against average axial, mean and perpendicular diffusivity under the significant voxels. The boxplots stand for mean, 95\% confidence interval and range. Outliers are depicted with "o".

\section{Study 2}

\section{Group independent component analysis}

MELODIC decomposed the participants' temporally concatenated data into 30 group average independent components, from which we retained components corresponding to the default mode, dorsal attention and salience networks (see Figure 3 for the spatial maps of said components). For assessing intrinsic connectivity in the salience network, we divided the respective component spatial map into 5 regions of interest (ROIs) using FSL's cluster tool. The ROIs included in further analyses were the bilateral anterior insulae (rAI and 1AI), the bilateral dorsolateral prefrontal cortices (rPFC and $\mathrm{PFC}$ ) and the dorsal anterior cingulate cortex (dACC). These group average ROI masks were used to extract regional time series for each individual. 

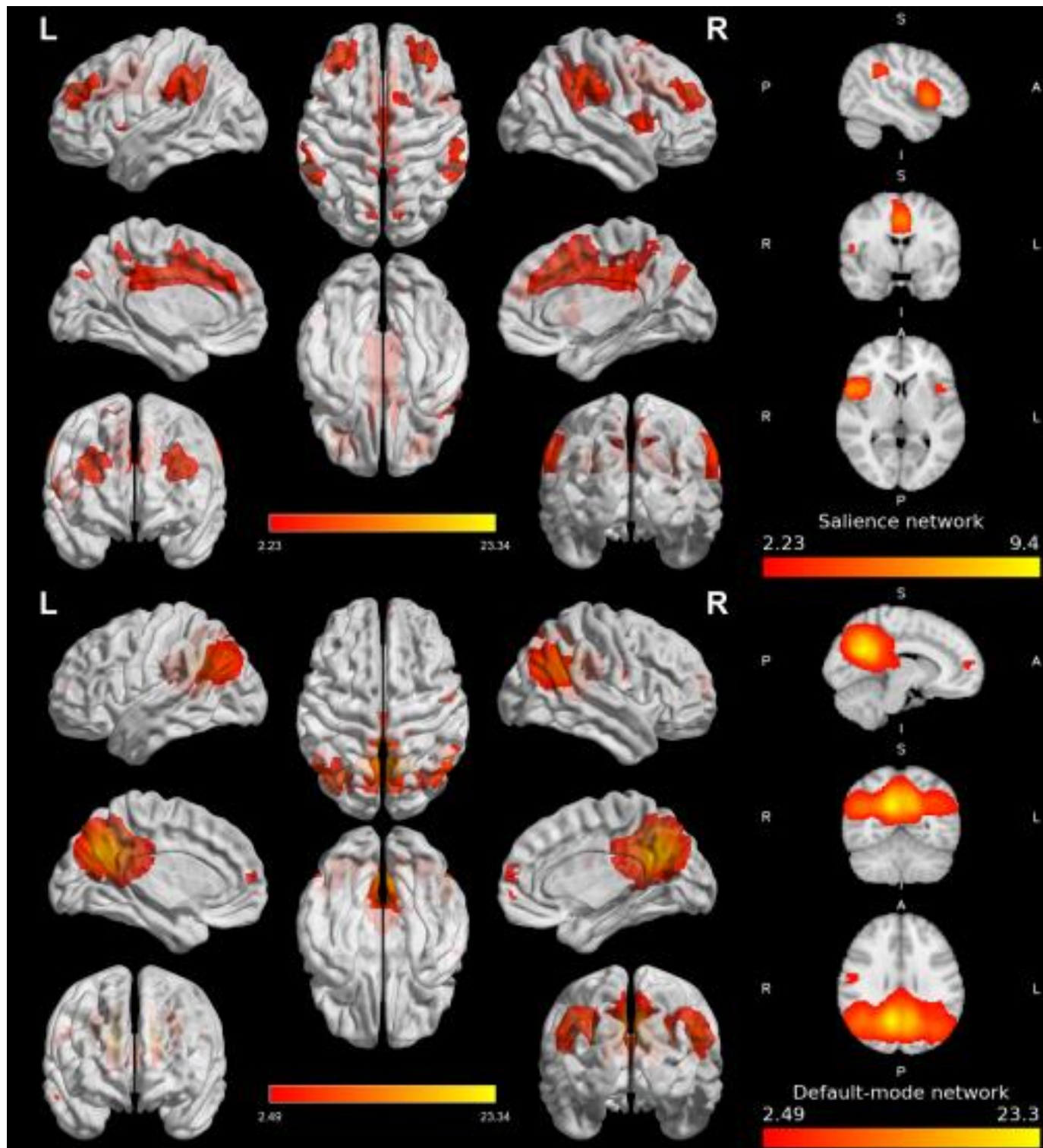

$\mathbf{R}$
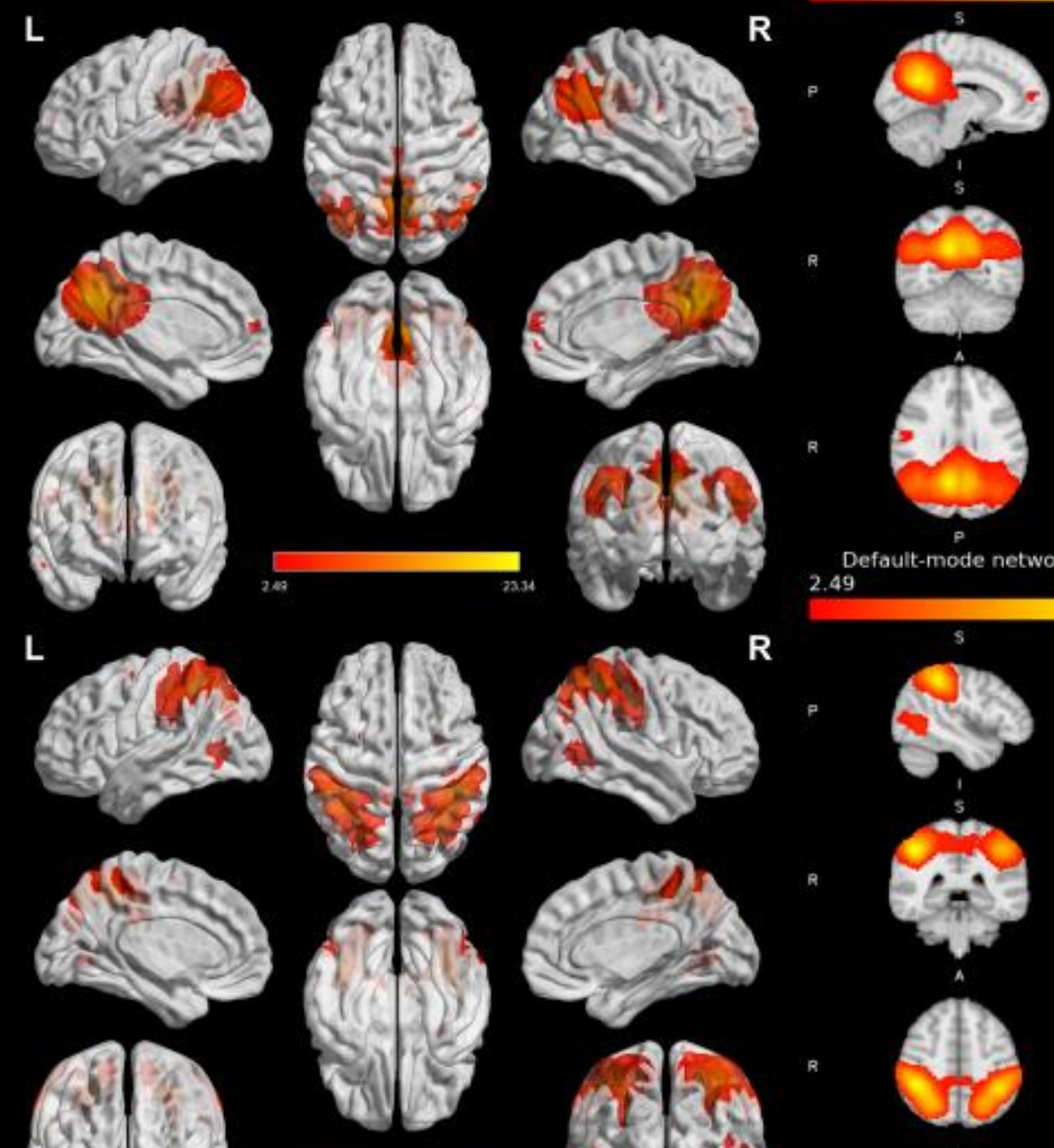

$\mathbf{R}$

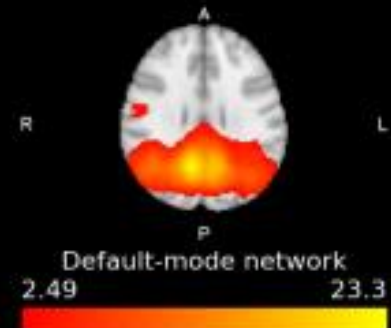

$\mathbf{R}$
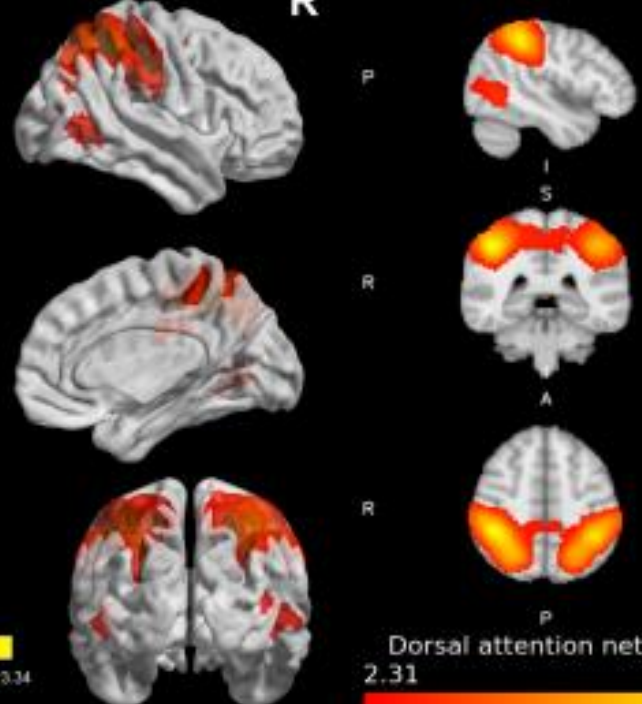

Dorsal attention network 2.31 
Figure 2: Results of the group-level independent component analysis. Thresholded independent component maps depicting the salience, default mode and dorsal attention networks were overlaid on a standard MNI brain template ( $2 \mathrm{~mm}$ resolution). 3D maps were created using BrainNet Viewer [49]. Color bars represent Z-values. 2D maps depict slices that contain the voxel with maximum Z-value. MNI-coordinates of these voxels are:

- Salience network: $\mathrm{x}=46 \mathrm{y}=-2 \mathrm{z}=4$

- Default mode network: $\mathrm{x}=10 \mathrm{y}=-58 \mathrm{z}=28$

- Dorsal attention network: $\mathrm{x}=46 \mathrm{y}=-38 \mathrm{z}=48$

\section{Temporal characteristics of connectivity in the salience network}

The variance of correlation was higher in the aura group compared to migraine without aura and healthy controls between the rAI and $\mathrm{dACC}(\mathrm{p}<0.011$ and $\mathrm{p}<0.026)$ and also higher in the aura group compared to healthy controls between the IPFC and AACC ( $\mathrm{p}<0.021)$. Temporally averaged correlation was lower in the migraine without aura group compared to migraine with aura and healthy controls between the rAI and rPFC ( $p<0.038$ and $p<0.037)$. For a summary of dynamic conditional correlation results, see Figure 4.

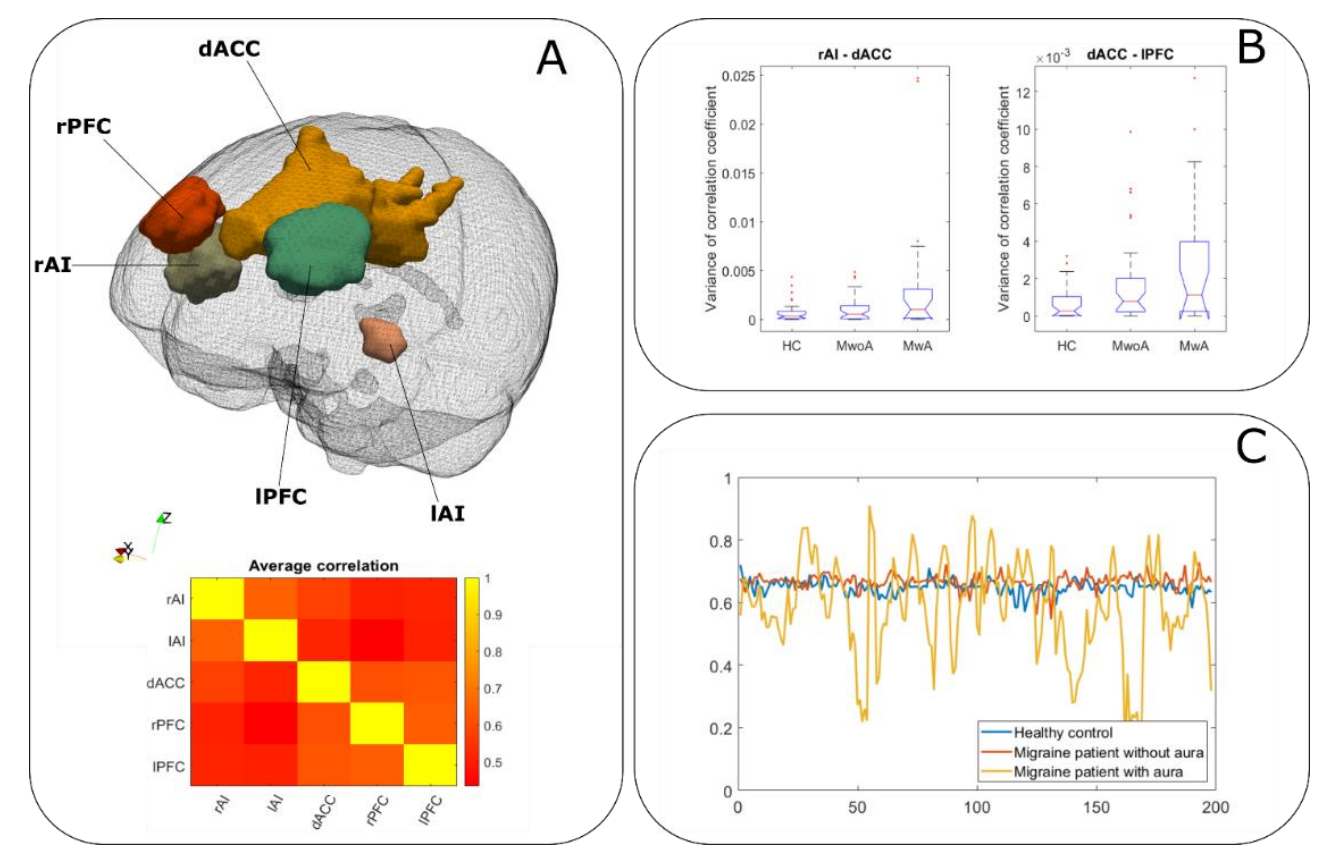

Figure 4: Temporal properties of connectivity within the salience network. (A) Regions of interest in the salience network, overlaid on a glass brain. The correlation matrix depicts the 
temporal average of pairwise dynamic conditional correlation between regions of interest. Abbreviations: r/lAI - right/left anterior insula, r/lPFC - right/left PFC, dACC - dorsal anterior cingulate cortex. (B) Boxplots depict significant differences of dynamic conditional correlation variance between groups in the salience network. The variability of correlation between the right anterior insula, dorsal anterior cingulate and left PFC was higher in migraine patients with aura, demonstrated by representative subjects on $(\mathbf{C})$.

\section{Causal interactions of the salience network and other large-scale networks}

The sum of causality from the salience to the dorsal attention network in the $0.01-0.05 \mathrm{~Hz}$ range was lower in migraine with aura compared to migraine without aura and healthy controls ( $<<0.032$ in both cases; see Figure 5). There was a trend of weaker causality from the default mode to the dorsal attention network in migraine with aura, which did not reach statistical significance.
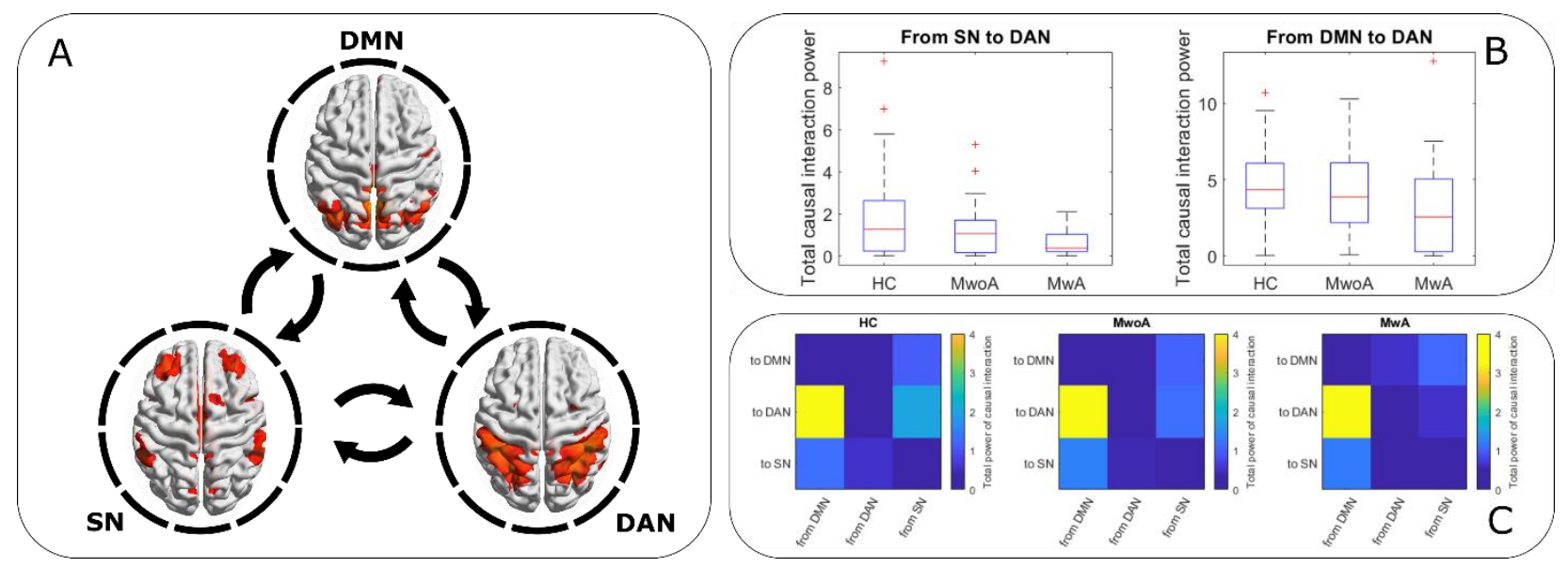

Figure 5: Causal interactions between large-scale resting-state networks. (A) Spectral Granger's causality was calculated between the salience, default mode and dorsal attention networks (SN, DMN and DAN, respectively). (B) Boxplots depict differences of sub- $0.5 \mathrm{~Hz}$ causal interactions between groups. Migraine patients with aura show significantly decreased information flow from the salience network to the dorsal attention network, and show a tendency of decreased information flow from the DMN to the DAN. (C) shows the full array of pairwise causal interaction strength between the investigated large-scale networks. 


\section{Relation to clinical variables}

We found no association between connectivity variance and clinical measures in the aura group. In migraine without aura, the variance of rPFC-1PFC and rAI-rPFC connectivity diminished with increasing attack frequency $(R=-0.516, p<0.003$ and $R=-0.456, p<0.012)$. Causal interaction power in the $0.01-0.05 \mathrm{~Hz}$ range between the default mode and dorsal attention networks diminished with longer disease duration in the migraine with aura group $(R=-0.525$, $\mathrm{p}<0.036)$. We also found a relationship between rPFC-dACC connectivity variance and salience-default mode network causality in the migraine with aura group $(R=-0.564, p<0.045)$.

\section{Discussion}

In the first study, we report significant correlation between diffusion parameters and interictal plasma PACAP38 in migraine patients in the occipital white matter. Similarly to our previous results [40], interictal PACAP38 was lower in the migraine group than in normal healthy controls.

The interpretation of diffusion tensor metrics in terms of microstructural characteristics is still open to debate. The fact that microstructural properties are described as a summation of tissue characteristics in voxels that are orders of magnitude larger than the actual nervous tissue structures complicates the issue further. However, it is generally accepted in the literature that lower FA or higher MD corresponds to a disrupted integrity of the white matter. AD and RD correlates with the number of axons and myelin integrity [141, 142]. In light of this, our results could be interpreted in a way that abnormally lower plasma PACAP38 levels in the interictal phase accompany higher axonal density and/or myelination in the optic radiation, corpus callosum and temporoparietal regions. The absence of correlation with FA may be due to the fact that FA, as a measure, combines $\mathrm{AD}$ and $\mathrm{RD}$ : a comparable change in both $\mathrm{AD}$ and $\mathrm{RD}$ would bring about FA changes only if the magnitude of change in the two perpendicular directions differs.

Since migraineurs often exhibit cortical hyperexcitability in the interictal term [143, 144], and this increased neuronal activity might also result in maladaptive plasticity (leading to more structured, compact white matter with the formation of more pronounced visual and painrelated pathways), our results could indicate a connection between PACAP-related signaling and such maladaptive remodelling related to migraine symptoms. In line with this, PACAP38- 
induced migraine-like attacks seem to bring about increased connectivity of the left visual cortex in the default mode network [43].

Naturally, there is a chance that the connection between PACAP38 levels and white matter integrity is coincidental. In this case, decreased interictal plasma PACAP38 levels might reflect changes in PACAP-ergic signalling that contribute to disrupted neurochemical coupling in migraine, which was previously reported by MRS studies [115]. In the long term, these functional alterations might lead to structural changes; longitudinal studies are required to understand how these connected alterations develop.

The spatial pattern of correlating diffusion characteristics suggests the involvement of the visual system, which shows widespread alterations in migraine [145]. Photophobia is a frequent accompanying symptom which occurs in a large portion of migraine patients. Also, bright light reportedly exacerbates headache intensity in migraine. Intrinsically photosensitive retinal ganglionic cells (ipRGCs; [146]) are implicated in the mechanisms underlying photophobia, and express PACAP38 as a co-transmitter in retino-hypothalamic projections [99, 147, 148]. Neurons in the suprachiasmatic nucleus, where these pathways are relayed, are connected lightsensitive neurons in the trigeminal nucleus caudalis [149], an area that also contains PACAP38expressing neurons [150]. These connections could provide a link between the visual and trigeminovascular system. IpRGCs also connect to pain centers in the thalamus in rats [151] and are considered parts of a photophobia pathway which allows for direct stimulation of trigeminal afferents through the eye without involving the optic nerve $[152,153]$.

Other animal studies found associations between PACAP38 and behavioural aspects of photophobia. After activation of the trigeminovascular system with nitroglycerol, PACAP38deficient mice show a lesser degree of light avoidance than wild-type mice [154]. Taking these findings into account, it is possible that reduced interictal PACAP38-levels are reflective of altered photophobia-associated signaling that co-occurs with altered white matter integrity of the optic tract. A previous study showing that PACAP38 uptake peaks in the occipital cortices after intranasal administration in mice provides further evidence for this [155].

In our study, the spatial pattern of correlation between microstructural properties and PACAP38-LI was predominantly left-sided. We have shown previously that subcortical structures exhibit asymmetry in microstructural characteristics [156], which might also be true 
in major white matter tracts [157]. Although not reaching significance when corrected for multiple comparisons, there were voxels in the contralateral white matter that mirror significant results at an uncorrected threshold of $\mathrm{p}<0.001$.

The change in the results' spatial pattern with the inclusion of disease duration as a covariate of no interest suggests that altered PACAP38 metabolism and its impact might develop as a function of disease progression, though it is unclear whether this is due to disease pathology or accumulating effects of chronic exposure to recurring headache attacks. In a different study we found that longer disease duration comes with decreased values of axial diffusivity in the left parietooccipital regions in migraine patients with aura [158], which appear in similar locations as PACAP38-related alterations in this current study. Since decreased PACAP38-levels and microstructural characteristics of the left thalamus correlate when correcting for disease duration, we suggest that PACAP38 might be associated with migraine-related white matter alterations in multiple ways, and this should be addressed in further research.

Our study has several limitations. Firstly, the patient population is heterogeneous as we included both migraine with and without aura patients. Our recent study demonstrated microstructural differences between the two subtypes of migraine [158], therefore, we repeated the analysis for migraine with and without aura patients separately. Since sample sises are smaller this way, no significant correlation was found in either group with interictal PACAP38. However, the uncorrected results depicted similar patterns of correlation in both groups, which indicates that possibly, there are no major differences in the connection between PACAP38-LI and diffusion characteristics. Still, studies with larger sample sises are needed to ascertain whether the two subgroups differ in PACAP38-metabolism.

Also, since PACAPergic signalling seems to be altered in migraine, in this study we decided to focus on the interindividual variation of PACAP38 in migraine patients. However, to our knowledge there is no information in the literature about the relationship between PACAP38 and white matter diffusion measures in healthy controls. Further, controlled studies should be conducted to compare the relationship between PACAP38-levels and microstructural characteristics in healthy controls and migraine patients.

In the second study, we showed that (i) migraineurs with aura exhibit more variable connectivity within the salience network, (ii) effective connectivity between the salience and 
dorsal attention resting-state networks is reduced in migraine with aura, (iii) and both alterations are connected to the patients' clinical condition and the variability of connectivity within the salience network. The main purpose of this work was to (I.) examine if the increased cortical excitability that is most prevalent in migraine with aura, results in an instability of intranetworks states in the salience network represented by the increased variance of interregional intrinsic dynamic correlations and (II.) describe whether the between-network interactions are affected by the salience network's intrinsic instability. We discuss these points further below.

The physiological mechanisms underlying dynamic functional connectivity have been the target of intensive research recently, which linked it to behavioural and cognitive phenomena [159], the power of the EEG signal in certain frequency bands [160], and showed that in certain diseases it carries meaningful information about the underlying processes. For example, in the case of temporal lobe epilepsy, dynamic functional connectivity showed an association with cortical hyperexcitability: networks related to seizure propagation (especially the midline cingulate areas) exhibited an elevated variance of connectivity strength which increased with longer disease duration [123]. In the current study, we showed that connectivity between the right anterior insula and dorsal anterior cingulate cortex (areas among core regions of the salience network [161]) is more variable in migraine patients with aura. This instability in connectivity might be rooted in several disease-related processes. (i) The salience network rates external stimuli as significant (salient) or insignificant to the person [161, 162]. Presumably, if hyperexcitable brain regions (like the visual cortex in migraine) provide more frequent input, this would result in adaptive changes, strengthening structural connections between salience network nodes. This plastic remodelling could allow for a greater range of synchronization (greater range of connectivity) within the salience network. Results from our previous study, in which we reported increased FA, lower MD and lower RD in various white matter tracts (including the cingular white matter) in migraine patients with aura compared to controls, provide evidence for this line of thought [158]. Similar changes in diffusion parameters are usually interpreted as a result of plastic reorganization in the white matter, which comes with more compact white matter microstructure [163]. Also, in the migraine without aura group, the degree of variability in salience network connections was lower than in migraine patients with aura, and lower variance of salience network connectivity correlated with higher attack frequency and longer disease duration. (ii) The increased temporal variability of connections 
within the salience network might be explained by the increased amounts of glutamate in the dACC reported in migraine patients [115], which might allow stimuli otherwise normally not deemed salient to elicit a heightened response. In line with this, functional connectivity of the dACC reportedly strengthens with elevated glutamate levels [164]. Salience network regions (especially the anterior insula and the dACC) also have important roles in pain processing [165], which suggests that more unstable connections in the network could be attributed to an increased allostatic burden that recurring migraine attacks pose. Our results, which show that the temporal variability of salience network connectivity differs between the two migraine subtypes might be interpreted as evidence to the contrary, although migraine patients without aura had significantly higher attack frequency in the study sample (two-tailed Student's t-test, $\mathrm{p}<0.01)$. Even so, temporal variability of salience network connectivity relates differently to attack frequency and disease duration in the two subtypes. Naturally, further research should be conducted to establish the pathophysiological processes underlying the more variable insuladACC connection in migraine.

Looking at large-scale network interactions, the salience network putatively helps in the transition from resting state (default mode) to active or task-related brain function [117, 118]. It is one of the networks implied in the three network model that provides the basis for the functional architecture that supports cognitive processes [166]. Alterations of functional and effective connectivity between the salience network and other large-scale networks have been previously reported in migraine patients [167]. In line with these reports, we found that information flow between the default mode, dorsal attention and salience networks is altered in migraine patients. Our results also link altered large-scale network interactions to an increased temporal variability of within-network connectivity in the salience network. An increase of variability in network connections might be a sign of a greater range of synchronization between network nodes, which would allow for more frequent signals to come from the anterior insula. This could in turn reduce the efficiency of further processing for stimuli that are deemed salient, and manifest as a change in large-scale network dynamics. We also found a tendency of diminished information flow from the default mode network to the salience network, possibly reflecting that the function of the salience network as a "switch" between the default mode and dorsal attention networks is harder to trigger in migraine patients, which could be a result of adaptation to a lower threshold for stimulus saliency in the face of an increased stimulus load 
brought on by alterations in sensory processing [168]. Higher attack frequency and longer disease duration was associated with reduced information flow between networks in the current study, which might provide some evidence for this hypothesis. However, further studies with paradigms that recruit saliency detection networks more consistently and accessibly are needed to investigate these possibilities.

This study also has several limitations. Firstly, since it is a fairly new area of investigation, the methodology of dynamic functional connectivity still evolves rapidly, bringing about a flurry of measures and metrics needing further evaluation, with no best one that is appropriate for every study design. In the current study, we use a metric that has been previously evaluated and deemed accurate and reproducible [135]. The temporal resolution of fMRI also restricts observation to the scale of seconds, therefore more information could be gained through the use of multiple modalities. Lastly, it is not yet fully established how dynamic changes observed during rest carry over to task performance; task designs that target saliency detection could also be more informative about disease-related changes.

\section{Conclusions}

Although various biomarkers derived from MRI measurements have proven informative and useful in migraine research, questions remain about the underlying pathophysiological mechanisms and how these markers can be integrated with other markers of the disease into a unified framework. In an effort to tie alterations detectable with MRI methods to neurochemical markers, we described a link between measures derived from diffusion-weighted MRI scans and serum levels of PACAP38, an important neuropeptide implicated in migraine pathogenesis. Placing migraine-specific MRI alterations in the context of neurochemical biomarkers of the disease emphasises the values of both markers and opens up new directions for future research. In the second study, using recent developments in the estimation of functional connectivity, we found that intrinsic connectivity is more variable in the salience network in migraine patients with aura compared to healthy controls and migraineurs without aura. Altered connectivity variance scaled with clinical parameters, and was associated with reduced interaction between large-scale networks. These results emphasise the importance of regarding the two main migraine subtypes separately, and add to our knowledge about changes in functional brain architecture that underlie disease processes in migraine. 


\section{Acknowledgements}

This work, although it ultimately carries the names of myself and my closest work affiliates, is a culmination of years of hard work done by a lot of remarkable people; work that was hard in different and sometimes subtle ways. I would like to dedicate this smaller-than-they-woulddeserve space to them.

Firstly, a special thank you goes to my supervisor, Zsigmond Tamás Kincses. I will always be grateful to him for encouraging me to keep working even when I wanted to orient my life in different directions. He is mainly responsible for kindling a scientific curiosity in me and remains an inspiration since I first lost track of ys and betas in his (otherwise very educational) explanation of general linear model theory.

Similarly, I would like to express my gratitude to my colleagues in the Neuroimaging Research Group, namely: Nikoletta Szabó, Péter Faragó, András Király, Eszter Tóth, Gergő Csete, Bence Bozsik, Krisztián Kocsis and Bálint Kincses. Thank you for being awesome and keeping a fledgling neuroimager interested and motivated. Further thanks are in order for the occasional motivational talks, shared lunches and billiard/bowling sessions.

Words are definitely not enough to express how thankful I am to my parents, siblings and family. Hopefully this work lives up to your support and company, to my father's homegrown tomatoes and chicken eggs, to my mother's excellent pizza recipe and morning coffee and to my siblings' annoyed looks when they realise I made most of the chocolate in the larder disappear.

Several medals should be given to my partner, Dóri, who endured and carried me through periods of demotivation and self-doubt. For this and many other things, I will always be thankful.

I also owe thanks to Professors László Vécsei and Péter Klivényi for giving me an opportunity to work at the Department of Neurology. Likewise, I would like to thank Bernadett Tuka and the Headache Research Group, staff members at the Departments of Neurology and Radiology for their constant, invaluable help and assistance with our research group's work. 


\section{References}

1. Olesen, J., International Classification of Headache Disorders. The Lancet Neurology, 2018. 17(5): p. 396-397.

2. Vanagaite, J., et al., Light-induced discomfort and pain in migraine. Cephalalgia, 1997. 17(7): p. 733-41.

3. Lauritzen, M., PATHOPHYSIOLOGY OF THE MIGRAINE AURA - THE SPREADING DEPRESSION THEORY. Brain, 1994. 117: p. 199-210.

4. Feigin, V.L., et al., Global, regional, and national burden of neurological disorders, 1990\&\#x2013;2016: a systematic analysis for the Global Burden of Disease Study 2016. The Lancet Neurology, 2019. 18(5): p. 459-480.

5. Yeh, W.Z., L. Blizzard, and B.V. Taylor, What is the actual prevalence of migraine? Brain and behavior, 2018. 8(6): p. e00950-e00950.

6. Bank, J. and S. Marton, Hungarian migraine epidemiology. Headache, 2000. 40(2): p. 164-9.

7. Goadsby, P.J., et al., Pathophysiology of Migraine: A Disorder of Sensory Processing. Physiol Rev, 2017. 97(2): p. 553-622.

8. Brennan, K.C. and D. Pietrobon, A Systems Neuroscience Approach to Migraine. Neuron, 2018. 97(5): p. 1004-1021.

9. Cevoli, S., V. Favoni, and P. Cortelli, Energy Metabolism Impairment in Migraine. Curr Med Chem, 2019. 26(34): p. 6253-6260.

10. Pietrobon, D. and M.A. Moskowitz, Pathophysiology of Migraine. Annual Review of Physiology, 2013. 75(1): p. 365-391.

11. Waeber, C. and M.A. Moskowitz, Migraine as an inflammatory disorder. Neurology, 2005. 64(10 Suppl 2): p. S9-15.

12. Levy, D., Migraine pain, meningeal inflammation, and mast cells. Curr Pain Headache Rep, 2009. 13(3): p. 237-40.

13. Tajti, J., et al., Migraine and neuropeptides. Neuropeptides, 2015. 52: p. 19-30.

14. Edvinsson, L., Innervation and effects of dilatory neuropeptides on cerebral vessels. Journal of Vascular Research, 1991. 28(1-3): p. 35-45.

15. Tajti, J., R. Uddman, and L. Edvinsson, Neuropeptide localization in the 'migraine generator'region of the human brainstem. Cephalalgia, 2001. 21(2): p. 96-101.

16. Uddman, R., et al., Neuropeptide expression in the human trigeminal nucleus caudalis and in the cervical spinal cord C1 and C2. Cephalalgia, 2002. 22(2): p. 112-116.

17. Weiller, C., et al., Brain stem activation in spontaneous human migraine attacks. Nature medicine, 1995. 1(7): p. 658-660.

18. Eftekhari, S. and L. Edvinsson, Calcitonin gene-related peptide (CGRP) and its receptor components in human and rat spinal trigeminal nucleus and spinal cord at C1-level. BMC neuroscience, 2011. 12(1): p. 1-18.

19. Thalakoti, S., et al., Neuron-glia signaling in trigeminal ganglion: implications for migraine pathology. Headache: The Journal of Head and Face Pain, 2007. 47(7): p. 1008-1023.

20. Edvinsson, L. and R. Uddman, Neurobiology in primary headaches. Brain research reviews, 2005. 48(3): p. 438-456.

21. Bigal, M.E., S. Walter, and A.M. Rapoport, Calcitonin gene-related peptide (CGRP) and migraine current understanding and state of development. Headache: The Journal of Head and Face Pain, 2013. 53(8): p. 1230-1244.

22. Strassman, A., S. Raymond, and R. Burstein, Sensitization of meningeal sensory neurons and the origin of headaches. Nature, 1996. 384(6609): p. 560-564. 
23. Juhasz, G., et al., NO-induced migraine attack: strong increase in plasma calcitonin generelated peptide (CGRP) concentration and negative correlation with platelet serotonin release. Pain, 2003. 106(3): p. 461-70.

24. Juhasz, G., et al., Sumatriptan Causes Parallel Decrease in Plasma Calcitonin Gene-Related Peptide (CGRP) Concentration and Migraine Headache During Nitroglycerin Induced Migraine Attack. Cephalalgia, 2005. 25(3): p. 179-183.

25. Hansen, J.M., et al., Calcitonin gene-related peptide triggers migraine-like attacks in patients with migraine with aura. Cephalalgia, 2010. 30(10): p. 1179-1186.

26. Edvinsson, L., et al., CGRP as the target of new migraine therapies - successful translation from bench to clinic. Nat Rev Neurol, 2018. 14(6): p. 338-350.

27. Hirabayashi, T., T. Nakamachi, and S. Shioda, Discovery of PACAP and its receptors in the brain. J Headache Pain, 2018. 19(1): p. 28.

28. Tuka, B., et al., Peripheral and central alterations of pituitary adenylate cyclase activating polypeptide-like immunoreactivity in the rat in response to activation of the trigeminovascular system. Peptides, 2012. 33(2): p. 307-316.

29. Nemeth, J., et al., Effect of pituitary adenylate cyclase activating polypeptide-38 on sensory neuropeptide release and neurogenic inflammation in rats and mice. Neuroscience, 2006. 143(1): p. 223-230.

30. Helyes, Z., et al., Inhibitory effect of PACAP-38 on acute neurogenic and non-neurogenic inflammatory processes in the rat. Peptides, 2007. 28(9): p. 1847-1855.

31. Sándor, K., et al., Divergent peripheral effects of pituitary adenylate cyclase-activating polypeptide-38 on nociception in rats and mice. PAIN ${ }^{\circledR}, 2009$. 141(1-2): p. 143-150.

32. Tajti, J., et al., Messenger molecules and receptor mRNA in the human trigeminal ganglion. Journal of the autonomic nervous system, 1999. 76(2-3): p. 176-183.

33. Palkovits, M., A. Somogyvári-Vigh, and A. Arimura, Concentrations of pituitary adenylate cyclase activating polypeptide (PACAP) in human brain nuclei. Brain research, 1995. 699(1): $p$. 116-120.

34. Légrádi, G., S. Shioda, and A. Arimura, Pituitary adenylate cyclase-activating polypeptide-like immunoreactivity in autonomic regulatory areas of the rat medulla oblongata. Neuroscience letters, 1994. 176(2): p. 193-196.

35. Koves, K. and A. Arimura, Go. rcs TG, SomogyvariVigh A: Comparative distribution of immunoreactive pituitary adenylate cyclase activating polypeptide and vasoactive intestinal polypeptide in rat forebrain. Neuroendocrinology, 1991. 54: p. 159-169.

36. Akerman, S. and P. Goadsby, VPAC1 and PAC1 receptor antagonists inhibit activation of the parasympathetic outflow to the cranial vasculature to prevent autonomic responses and neuronal firing in the trigeminocervical complex: PO310. Cephalalgia, 2009. 29.

37. Knutsson, M. and L. Edvinsson, Distribution of mRNA for VIP and PACAP receptors in human cerebral arteries and cranial ganglia. NeuroReport, 2002. 13(4): p. 507-509.

38. Kulka, M., et al., Neuropeptides activate human mast cell degranulation and chemokine production. Immunology, 2008. 123(3): p. 398-410.

39. Syed, A.U., et al., Pituitary adenylate cyclase-activating polypeptide (PACAP) potently dilates middle meningeal arteries: implications for migraine. Journal of Molecular Neuroscience, 2012. 48(3): p. 574-583.

40. Tuka, B., et al., Alterations in PACAP-38-like immunoreactivity in the plasma during ictal and interictal periods of migraine patients. Cephalalgia, 2013. 33(13): p. 1085-95.

41. Schytz, H.W., et al., PACAP38 induces migraine-like attacks in patients with migraine without aura. Brain, 2008. 132(1): p. 16-25. 
42. Amin, F.M., et al., Investigation of the pathophysiological mechanisms of migraine attacks induced by pituitary adenylate cyclase-activating polypeptide-38. Brain, 2014. 137(3): p. 779794.

43. Amin, F.M., et al., Change in brain network connectivity during PACAP38-induced migraine attacks: A resting-state functional MRI study. Neurology, 2016. 86(2): p. 180-7.

44. Markovics, A., et al., Pituitary adenylate cyclase-activating polypeptide plays a key role in nitroglycerol-induced trigeminovascular activation in mice. Neurobiology of disease, 2012. 45(1): p. 633-644.

45. Rubio-Beltrán, E., et al., PACAP38 and PAC1 receptor blockade: a new target for headache? The Journal of Headache and Pain, 2018. 19(1): p. 64.

46. Burstein, R., et al., Thalamic sensitization transforms localised pain into widespread allodynia. Ann Neurol, 2010. 68(1): p. 81-91.

47. Burstein, R., et al., Chemical stimulation of the intracranial dura induces enhanced responses to facial stimulation in brain stem trigeminal neurons. J Neurophysiol, 1998. 79(2): p. 964-82.

48. Pietrobon, D. and J. Striessnig, Neurobiology of migraine. Nat Rev Neurosci, 2003. 4(5): p. 386-98.

49. Lauritzen, M., Pathophysiology of the migraine aura. The spreading depression theory. Brain, 1994. 117 ( Pt 1): p. 199-210.

50. Abad, N., et al., Metabolic assessment of a migraine model using relaxation-enhanced (1) $\mathrm{H}$ spectroscopy at ultrahigh field. Magn Reson Med, 2018. 79(3): p. 1266-1275.

51. Sándor, P.S., et al., MR-spectroscopic imaging during visual stimulation in subgroups of migraine with aura. Cephalalgia, 2005. 25(7): p. 507-18.

52. Sarchielli, $\mathrm{P}$., et al., Functional $1 H-M R S$ findings in migraine patients with and without aura assessed interictally. Neuroimage, 2005. 24(4): p. 1025-31.

53. Dehghan, A., et al., Neurochemical metabolite alterations of the occipital lobe in migraine without aura by proton magnetic resonance spectroscopy. The Neuroradiology Journal, 2020: p. 1971400920932793.

54. Szabó, N., et al., White matter microstructural alterations in migraine: A diffusion-weighted MRI study. PAIN, 2012. 153(3): p. 651-656.

55. Szabó, N., et al., Evidence for Plastic Processes in Migraine with Aura: A Diffusion Weighted MRI Study. Front Neuroanat, 2017. 11: p. 138.

56. Faragó, P., et al., Interictal brain activity differs in migraine with and without aura: resting state fMRI study. J Headache Pain, 2017. 18(1): p. 8.

57. Chong, C.D., T.J. Schwedt, and A. Hougaard, Brain functional connectivity in headache disorders: A narrative review of MRI investigations. J Cereb Blood Flow Metab, 2019. 39(4): p. 650-669.

58. Bashir, A., et al., Migraine and structural changes in the brain: a systematic review and metaanalysis. Neurology, 2013. 81(14): p. 1260-8.

59. Komáromy, H., et al., Influence of hemispheric white matter lesions and migraine characteristics on cortical thickness and volume. J Headache Pain, 2019. 20(1): p. 4.

60. Kim, J.H., et al., Regional grey matter changes in patients with migraine: a voxel-based morphometry study. Cephalalgia, 2008. 28(6): p. 598-604.

61. Schmidt-Wilcke, T., et al., Subtle grey matter changes between migraine patients and healthy controls. Cephalalgia, 2008. 28(1): p. 1-4.

62. Valfrè, W., et al., Voxel-based morphometry reveals gray matter abnormalities in migraine. Headache, 2008. 48(1): p. 109-17.

63. Messina, R., et al., Cortical abnormalities in patients with migraine: a surface-based analysis. Radiology, 2013. 268(1): p. 170-80. 
64. Rodriguez-Raecke, R., et al., Brain gray matter decrease in chronic pain is the consequence and not the cause of pain. J Neurosci, 2009. 29(44): p. 13746-50.

65. Rodriguez-Raecke, R., et al., Structural brain changes in chronic pain reflect probably neither damage nor atrophy. PLoS One, 2013. 8(2): p. e54475.

66. Schwedt, T.J., et al., Migraine Subclassification via a Data-Driven Automated Approach Using Multimodality Factor Mixture Modeling of Brain Structure Measurements. Headache, 2017. 57(7): p. 1051-1064.

67. Hougaard, A., et al., Sensory migraine aura is not associated with structural grey matter abnormalities. Neuroimage Clin, 2016. 11: p. 322-327.

68. Hougaard, A., F.M. Amin, and M. Ashina, Migraine and structural abnormalities in the brain. Curr Opin Neurol, 2014. 27(3): p. 309-14.

69. Hadjikhani, N., et al., Mechanisms of migraine aura revealed by functional MRI in human visual cortex. Proc Natl Acad Sci U S A, 2001. 98(8): p. 4687-92.

70. Gaist, D., et al., Migraine with visual aura associated with thicker visual cortex. Brain, 2018. 141(3): p. 776-785.

71. Burke, M.J., et al., Mapping migraine to a common brain network. Brain, 2020. 143(2): p. 541-553.

72. Kruit, M.C., et al., Migraine is associated with an increased risk of deep white matter lesions, subclinical posterior circulation infarcts and brain iron accumulation: the population-based MRI CAMERA study. Cephalalgia, 2010. 30(2): p. 129-36.

73. Kruit, M.C., et al., Migraine as a risk factor for subclinical brain lesions. Jama, 2004. 291(4): p. 427-34.

74. Gursoy-Ozdemir, Y., et al., Cortical spreading depression activates and upregulates MMP-9. J Clin Invest, 2004. 113(10): p. 1447-55.

75. Basser, P.J., J. Mattiello, and D. LeBihan, MR diffusion tensor spectroscopy and imaging. Biophys J, 1994. 66(1): p. 259-67.

76. Chong, C.D. and T.J. Schwedt, Migraine affects white-matter tract integrity: A diffusion-tensor imaging study. Cephalalgia, 2015. 35(13): p. 1162-71.

77. Rocca, M.A., et al., Selective diffusion changes of the visual pathways in patients with migraine: a 3-T tractography study. Cephalalgia, 2008. 28(10): p. 1061-8.

78. DaSilva, A.F., et al., Interictal alterations of the trigeminal somatosensory pathway and periaqueductal gray matter in migraine. Neuroreport, 2007. 18(4): p. 301-5.

79. Granziera, C., et al., Anatomical alterations of the visual motion processing network in migraine with and without aura. PLoS Med, 2006. 3(10): p. e402.

80. Xie, H., et al., Right-to-left shunt may be prone to affect the white matter integrity of posterior circulation in migraine without aura. Neurol Sci, 2018. 39(1): p. 119-125.

81. Davis, K.D., et al., Discovery and validation of biomarkers to aid the development of safe and effective pain therapeutics: challenges and opportunities. Nature Reviews Neurology, 2020. 16(7): p. 381-400.

82. Hougaard, A., et al., Increased intrinsic brain connectivity between pons and somatosensory cortex during attacks of migraine with aura. Hum Brain Mapp, 2017. 38(5): p. 2635-2642.

83. Coppola, G., et al., Thalamo-cortical network activity during spontaneous migraine attacks. Neurology, 2016. 87(20): p. 2154-2160.

84. Gil-Gouveia, R., A.G. Oliveira, and I.P. Martins, Cognitive dysfunction during migraine attacks: a study on migraine without aura. Cephalalgia, 2015. 35(8): p. 662-74.

85. Conforto, A.B., et al., Interictal abnormal fMRI activation of visual areas during a motor task cued by visual stimuli in migraine. Einstein (Sao Paulo), 2017. 15(1): p. 17-23. 
86. Ren, J., et al., Abnormal functional connectivity under somatosensory stimulation in migraine: a multi-frequency magnetoencephalography study. J Headache Pain, 2019. 20(1): p. 3.

87. Brighina, F., et al., Modulation of visual cortical excitability in migraine with aura: effects of 1 $\mathrm{Hz}$ repetitive transcranial magnetic stimulation. Exp Brain Res, 2002. 145(2): p. 177-81.

88. Antal, A., et al., Altered motion perception in migraineurs: evidence for interictal cortical hyperexcitability. Cephalalgia, 2005. 25(10): p. 788-794.

89. Hadjikhani, N., et al., The missing link: enhanced functional connectivity between amygdala and visceroceptive cortex in migraine. Cephalalgia, 2013. 33(15): p. 1264-8.

90. Zhang, J., et al., The sensorimotor network dysfunction in migraineurs without aura: a restingstate fMRI study. J Neurol, 2017. 264(4): p. 654-663.

91. Tessitore, A., et al., Abnormal Connectivity Within Executive Resting-State Network in Migraine With Aura. Headache, 2015. 55(6): p. 794-805.

92. Russo, A., et al., Executive resting-state network connectivity in migraine without aura. Cephalalgia, 2012. 32(14): p. 1041-8.

93. Tedeschi, G., et al., Increased interictal visual network connectivity in patients with migraine with aura. Cephalalgia, 2016. 36(2): p. 139-47.

94. Niddam, D.M., et al., Reduced functional connectivity between salience and visual networks in migraine with aura. Cephalalgia, 2016. 36(1): p. 53-66.

95. Hougaard, A., et al., No abnormalities of intrinsic brain connectivity in the interictal phase of migraine with aura. Eur J Neurol, 2015. 22(4): p. 702-e46.

96. Banks, W.A., et al., Passage of pituitary adenylate cyclase activating polypeptide1-27 and pituitary adenylate cyclase activating polypeptide1-38 across the blood-brain barrier. J Pharmacol Exp Ther, 1993. 267(2): p. 690-6.

97. Amin, F.M. and H.W. Schytz, Transport of the pituitary adenylate cyclase-activating polypeptide across the blood-brain barrier: implications for migraine. J Headache Pain, 2018. 19(1): p. 35.

98. Zhang, Y., et al., The effect of pituitary adenylate cyclase activating peptide (PACAP) on the nociceptive formalin test. Neurosci Lett, 1996. 207(3): p. 187-90.

99. Engelund, A., et al., Vesicular glutamate transporter 2 (VGLUT2) is co-stored with PACAP in projections from the rat melanopsin-containing retinal ganglion cells. Cell Tissue Res, 2010. 340(2): p. 243-55.

100. Guo, S., et al., Part II: Biochemical changes after pituitary adenylate cyclase-activating polypeptide-38 infusion in migraine patients. Cephalalgia, 2017. 37(2): p. 136-147.

101. Yilmaz, N., et al., Elevated S10OB and Neuron Specific Enolase Levels in Patients with Migraine-without Aura: Evidence for Neurodegeneration? Cell Mol Neurobiol, 2011. Epub ahead of print.

102. Ogata, K., et al., PACAP enhances axon outgrowth in cultured hippocampal neurons to a comparable extent as BDNF. PLoS One, 2015. 10(3): p. e0120526.

103. Shioda, S. and T. Nakamachi, PACAP as a neuroprotective factor in ischemic neuronal injuries. Peptides, 2015. 72: p. 202-7.

104. Hutchison, R.M., et al., Dynamic functional connectivity: promise, issues, and interpretations. Neuroimage, 2013. 80: p. 360-78.

105. Chang, C. and G.H. Glover, Time-frequency dynamics of resting-state brain connectivity measured with fMRI. Neuroimage, 2010. 50(1): p. 81-98.

106. Poldrack, R.A., et al., Long-term neural and physiological phenotyping of a single human. Nat Commun, 2015. 6: p. 8885.

107. Du, Y., et al., Dynamic functional connectivity impairments in early schizophrenia and clinical high-risk for psychosis. Neuroimage, 2018. 180(Pt B): p. 632-645. 
108. Falahpour, M., et al., Underconnected, But Not Broken? Dynamic Functional Connectivity MRI Shows Underconnectivity in Autism Is Linked to Increased Intra-Individual Variability Across Time. Brain Connect, 2016. 6(5): p. 403-14.

109. Schumacher, J., et al., Dynamic functional connectivity changes in dementia with Lewy bodies and Alzheimer's disease. Neurolmage: Clinical, 2019. 22: p. 101812.

110. Díez-Cirarda, M., et al., Dynamic functional connectivity in Parkinson's disease patients with mild cognitive impairment and normal cognition. Neurolmage: Clinical, 2018. 17: p. 847-855.

111. d'Ambrosio, A., et al., Reduced dynamics of functional connectivity and cognitive impairment in multiple sclerosis. Mult Scler, 2020. 26(4): p. 476-488.

112. Filippi, M., et al., Resting State Dynamic Functional Connectivity in Neurodegenerative Conditions: A Review of Magnetic Resonance Imaging Findings. Front Neurosci, 2019. 13: p. 657.

113. Tu, Y., et al., Abnormal thalamocortical network dynamics in migraine. Neurology, 2019. 92(23): p. e2706-e2716.

114. Lee, M.J., et al., Dynamic functional connectivity of the migraine brain: a resting-state functional magnetic resonance imaging study. PAIN, 2019. 160(12): p. 2776-2786.

115. Younis, S., et al., Migraine and magnetic resonance spectroscopy: a systematic review. Curr Opin Neurol, 2017. 30(3): p. 246-262.

116. Charles, A.C. and S.M. Baca, Cortical spreading depression and migraine. Nat Rev Neurol, 2013. 9(11): p. 637-44.

117. Li, S., et al., The Hierarchical Organization of the Default, Dorsal Attention and Salience Networks in Adolescents and Young Adults. Cerebral Cortex, 2017. 28(2): p. 726-737.

118. Sridharan, D., D.J. Levitin, and V. Menon, A critical role for the right fronto-insular cortex in switching between central-executive and default mode networks. Proc Natl Acad Sci U S A, 2008. 105(34): p. 12569-74.

119. Chand, G.B. and M. Dhamala, Interactions Among the Brain Default mode, Salience, and Central-Executive Networks During Perceptual Decision-Making of Moving Dots. Brain Connect, 2016. 6(3): p. 249-54.

120. Antal, A., et al., Altered motion perception in migraineurs: evidence for interictal cortical hyperexcitability. Cephalalgia, 2005. 25(10): p. 788-94.

121. Zarcone, D. and S. Corbetta, Shared mechanisms of epilepsy, migraine and affective disorders. Neurol Sci, 2017. 38(Suppl 1): p. 73-76.

122. Mantegazza, M. and S. Cestele, Pathophysiological mechanisms of migraine and epilepsy: Similarities and differences. Neurosci Lett, 2018. 667: p. 92-102.

123. Morgan, V.L., B. Abou-Khalil, and B.P. Rogers, Evolution of functional connectivity of brain networks and their dynamic interaction in temporal lobe epilepsy. Brain Connect, 2015. 5(1): p. 35-44.

124. Luo, C., et al., Altered intrinsic functional connectivity of the salience network in childhood absence epilepsy. J Neurol Sci, 2014. 339(1-2): p. 189-95.

125. Smith, S.M., et al., Advances in functional and structural MR image analysis and implementation as FSL. Neuroimage, 2004. 23 Suppl 1: p. S208-19.

126. Smith, S.M., et al., Tract-based spatial statistics: voxelwise analysis of multi-subject diffusion data. Neuroimage, 2006. 31(4): p. 1487-505.

127. Winkler, A.M., et al., Permutation inference for the general linear model. Neurolmage, 2014. 92: p. 381-397.

128. Smith, S.M. and T.E. Nichols, Threshold-free cluster enhancement: addressing problems of smoothing, threshold dependence and localisation in cluster inference. Neuroimage, 2009. 44(1): p. 83-98. 
129. Jenkinson, M., et al., Improved optimization for the robust and accurate linear registration and motion correction of brain images. Neuroimage, 2002. 17(2): p. 825-41.

130. Smith, S.M., Fast robust automated brain extraction. Hum Brain Mapp, 2002. 17(3): p. 14355.

131. Beckmann, C.F. and S.M. Smith, Probabilistic independent component analysis for functional magnetic resonance imaging. IEEE Trans Med Imaging, 2004. 23(2): p. 137-52.

132. Griffanti, L., et al., Hand classification of fMRI ICA noise components. Neuroimage, 2017. 154: p. 188-205.

133. Nickerson, L.D., et al., Using Dual Regression to Investigate Network Shape and Amplitude in Functional Connectivity Analyses. Front Neurosci, 2017. 11: p. 115.

134. Lindquist, M.A., et al., Evaluating dynamic bivariate correlations in resting-state fMRI: a comparison study and a new approach. Neuroimage, 2014. 101: p. 531-46.

135. Choe, A.S., et al., Comparing test-retest reliability of dynamic functional connectivity methods. Neuroimage, 2017. 158: p. 155-175.

136. Engle, R.F., Autoregressive Conditional Heteroscedasticity with Estimates of the Variance of United Kingdom Inflation. Econometrica, 1982. 50(4): p. 987-1007.

137. Granger, C.W.J., Investigating Causal Relations by Econometric Models and Cross-spectral Methods. Econometrica, 1969. 37(3): p. 424-438.

138. Seth, A.K., A.B. Barrett, and L. Barnett, Granger causality analysis in neuroscience and neuroimaging. J Neurosci, 2015. 35(8): p. 3293-7.

139. Wen, X., G. Rangarajan, and M. Ding, Is Granger causality a viable technique for analyzing fMRI data? PloS one, 2013. 8(7): p. e67428-e67428.

140. Kaminski, M., et al., Evaluating causal relations in neural systems: granger causality, directed transfer function and statistical assessment of significance. Biol Cybern, 2001. 85(2): p. 14557.

141. Song, S.K., et al., Demyelination increases radial diffusivity in corpus callosum of mouse brain. Neuroimage, 2005. 26(1): p. 132-40.

142. Senda, J., et al., MRI mean diffusivity detects widespread brain degeneration in multiple sclerosis. Journal of the Neurological Sciences, 2012. 319(1-2): p. 105-110.

143. Afra, J., et al., Comparison of visual and auditory evoked cortical potentials in migraine patients between attacks. Clin Neurophysiol, 2000. 111(6): p. 1124-9.

144. Farago, P., et al., Interictal brain activity differs in migraine with and without aura: resting state fMRI study. J Headache Pain, 2017. 18(1): p. 8.

145. Kowacs, P.A., M.A. Utiumi, and E.J. Piovesan, The visual system in migraine: from the bench side to the office. Headache, 2015. 55 Suppl 1: p. 84-98.

146. Noseda, R., et al., A neural mechanism for exacerbation of headache by light. Nat Neurosci, 2010. 13(2): p. 239-45.

147. Hannibal, J., et al., Melanopsin is expressed in PACAP-containing retinal ganglion cells of the human retinohypothalamic tract. Invest Ophthalmol Vis Sci, 2004. 45(11): p. 4202-9.

148. Hannibal, J., et al., Pituitary adenylate cyclase-activating peptide (PACAP) in the retinohypothalamic tract: a potential daytime regulator of the biological clock. J Neurosci, 1997. 17(7): p. 2637-44.

149. Okamoto, K., et al., Bright light activates a trigeminal nociceptive pathway. Pain, 2010. 149(2): p. 235-42.

150. Uddman, R., et al., Neuropeptide expression in the human trigeminal nucleus caudalis and in the cervical spinal cord C1 and C2. Cephalalgia, 2002. 22(2): p. 112-6.

151. Noseda, R. and R. Burstein, Advances in understanding the mechanisms of migraine-type photophobia. Curr Opin Neurol, 2011. 24(3): p. 197-202. 
152. Amini, A., K. Digre, and W.T. Couldwell, Photophobia in a blind patient: An alternate visual pathway. Case report. J Neurosurg, 2006. 105(5): p. 765-8.

153. Dolgonos, S., H. Ayyala, and C. Evinger, Light-induced trigeminal sensitization without central visual pathways: another mechanism for photophobia. Invest Ophthalmol Vis Sci, 2011. 52(11): p. 7852-8.

154. Markovics, A., et al., Pituitary adenylate cyclase-activating polypeptide plays a key role in nitroglycerol-induced trigeminovascular activation in mice. Neurobiol Dis, 2012. 45(1): p. 63344.

155. Nonaka, N., et al., Intranasal administration of PACAP: uptake by brain and regional brain targeting with cyclodextrins. Peptides, 2012. 36(2): p. 168-75.

156. Kiraly, A., et al., Macro- and microstructural alterations of the subcortical structures in episodic cluster headache. Cephalalgia, 2017: p. 333102417703762.

157. Wahl, M., et al., Microstructural correlations of white matter tracts in the human brain. Neuroimage, 2010. 51(2): p. 531-41.

158. Szabo, N., et al., Evidence for Plastic Processes in Migraine with Aura: A Diffusion Weighted MRI Study. Front Neuroanat, 2017. 11: p. 138.

159. Cohen, J.R., The behavioral and cognitive relevance of time-varying, dynamic changes in functional connectivity. Neurolmage, 2018. 180: p. 515-525.

160. Tagliazucchi, E., et al., Dynamic BOLD functional connectivity in humans and its electrophysiological correlates. Front Hum Neurosci, 2012. 6: p. 339.

161. Menon, V. and L.Q. Uddin, Saliency, switching, attention and control: a network model of insula function. Brain Struct Funct, 2010. 214(5-6): p. 655-67.

162. Sridharan, D., D.J. Levitin, and V. Menon, A critical role for the right fronto-insular cortex in switching between central-executive and default mode networks. Proceedings of the National Academy of Sciences, 2008. 105(34): p. 12569-12574.

163. Scholz, J., et al., Training induces changes in white-matter architecture. Nat Neurosci, 2009. 12(11): p. 1370-1.

164. Levar, N., et al., Anterior cingulate GABA and glutamate concentrations are associated with resting-state network connectivity. Scientific Reports, 2019. 9(1): p. 2116.

165. Legrain, V., et al., The pain matrix reloaded: A salience detection system for the body. Progress in Neurobiology, 2011. 93(1): p. 111-124.

166. Jiang, T., Brainnetome: a new -ome to understand the brain and its disorders. Neuroimage, 2013. 80: p. 263-72.

167. Yu, D., et al., Abnormal functional integration across core brain networks in migraine without aura. Mol Pain, 2017. 13: p. 1744806917737461.

168. Peng, K.P. and A. May, Migraine understood as a sensory threshold disease. Pain, 2019. 160(7): p. 1494-1501. 